\title{
Fully Dispersive Models for Moving Loads on Ice Sheets
}

\author{
E. Dinvay ${ }^{1}$, H. Kalisch ${ }^{1} \dagger$ and E. I. Pără ${ }^{2}$ \\ ${ }^{1}$ Department of Mathematics, University of Bergen, Postbox 7800, 5020 Bergen, Norway \\ ${ }^{2}$ School of Mathematics, University of East Anglia, Norwich Research Park, Norwich, NR4 \\ $7 \mathrm{TJ}, \mathrm{UK}$
}

(Received xx; revised xx; accepted $\mathrm{xx}$ )

The response of a floating elastic plate to the motion of a moving load is studied using a fully dispersive weakly nonlinear system of equations. The system allows for accurate description of waves across the whole spectrum of wavelengths and also incorporates nonlinearity, forcing and damping. The flexural-gravity waves described by the system are time-dependent responses to a forcing with a described weight distribution, moving at a time-dependent velocity. The model is versatile enough to allow the study of a wide range of situations including the motion of a combination of point loads and loads of arbitrary shape.

Numerical solutions of the system are compared to data from a number of field campaigns on ice-covered lakes, and good agreement between the deflectometer records and the numerical simulations is observed in most cases. Consideration is also given to waves generated by an accelerating or decelerating load, and it is shown that a decelerating load may trigger a wave response with a far greater amplitude than a load moving at constant celerity.

\section{Key words:}

\section{Introduction}

Flexural-gravity waves occur naturally in ice sheets floating on various bodies of water in cold regions, and the study of such waves has a long history. The pace of scientific inquiries into the nature of flexural-gravity waves intensified in the middle of the 20th century, prompted by the increasing use of solid ice covers to support mechanized transportation systems. In Canada for example, ice-covered lakes enabled the routing of trucks on winter roads built partially on ice, and in some places air strips and train tracks were built on thick ice covers. As these endeavors met with varied success, sometimes resulting in loss of life and equipment, it became clear that there was a need to improve our understanding of engineering properties of ice covers such as bearing capacity, resonant behavior, and the susceptibility to crack formation.

A number of experimental campaigns were mounted with the goal of understanding the wave response to moving loads on ice covers. Some of these studies, such as Takizawa (1987) and Wilson (1955) also included mathematical treatments based on linear wave theory, and these combined efforts gave some information about possible resonances and critical load speeds. In addition, efforts were undertaken to develop more sophisticated

$\dagger$ Email address for correspondence: henrik.kalisch@uib.no 
mathematical models that could predict the wave response to a moving load. Prompted by observed oscillations in the ice cover at McMurdo Sound (Antarctica) during the approach and landing of transport aircraft a considerable distance away, Davys, Hosking \& Sneyd (1985) used classical techniques of contour integration to evaluate the integrals appearing in the steady formulation of the hydro-elastic wave problem with a point-load, and found intricate wave patterns including caustics and zones of zero wave response. In a spectacular contribution, it was recently shown by Babaei, Van der Sanden, Short \& Barrette (2016) and Van der Sanden \& Short (2017) that such wave patterns can actually be found in the field by using satellite SAR radar observations of an ice sheet during the motion of a vehicle.

The analysis pioneered in Davys, Hosking \& Sneyd (1985) has been used and extended by a number of authors, but the main restrictions of a point load and constant load speed have only been partially removed in subsequent works. While the use of a point load as forcing is unproblematic due to the fact that the shortest waves created by the load are generally still very much longer than the typical load itself, the adherence to constant load speed in most works on the subject is somewhat more restrictive. Indeed, the issue of variable load speed was already raised by Beltaos (1981), but as far as we know this issue has only been investigated in very few contributions. The authors of Miles \& Sneyd (2003) showed that the wave response to an accelerating load stays bounded; the landing and deceleration of an airplane on ice cover was investigated in Matiushina, Pogorelova \& Kozin (2016), and the wave resistance due to the unsteady motion of and air-cushion vehicle on a supporting ice sheet was studied in Pogorelova (2008).

The necessity of making allowances for time-dependency is already implicit in the works of Davys, Hosking \& Sneyd (1985); Kheysin (1971); Schulkes \& Sneyd (1988) where it was found in that in addition to transient effects due to an impulsively started load, some constant load speeds lead to genuinely time-dependent wave responses. In particular, these authors showed that if the load is moving at the critical speed $V=c_{\min }$ which is the minimum of the dispersion curve defined in (1.2), the wave amplitude grows as $t^{1 / 2}$, where $t$ is the time variable. Schulkes \& Sneyd (1988) investigated moving line loads and characterized a second critical load speed $V=\sqrt{g H}$, where $g$ is the gravitational acceleration and $H$ is the depth of the fluid. While the analysis of one-dimensional wave patterns of Schulkes \& Sneyd (1988) showed an amplitude growth of $t^{1 / 3}$, the work of Milinazzo, Shinbrot \& Evans (1995) and Nugroho, Wang, Hosking \& Milinazzo (1999) implied that the amplitude of a two-dimensional wave response to a point-load moving at the speed $V=\sqrt{g H}$ is bounded.

The experiments conducted by Wilson (1955) at Mille Lacs in Minnesota, USA uncovered the existence of a time-lag between the passage of the load and the greatest depression of the ice sheet. The in-depth field campaign coordinated by Takizawa, and described in detail in Takizawa $(1978,1985,1987,1988)$ confirmed the existence of the time-lag between the passage of the load and the greatest depression of the ice sheet. In Takizawa $(1987,1988)$, a linear ordinary differential equation featuring a simple damping term was put forward, and this model was able to predict a time-lag such as observed in the field measurements. However, since this model was also based on the assumption of steady-state responses, no time-dependent wave solutions could be described.

While damping has the benefit of allowing a time-lag between the passage of the load and the maximum depression of the ice sheet, nonlinearity may also be important to describe the response exhibited near the critical load speed $V=c_{m i n}$. Părău \& Dias (2002) included nonlinearity in reconsidering the two resonances (singularities) inherent in previous linear elastic theory for a line load such as studied by Kheysin (1963) and Schulkes \& Sneyd (1988), and demonstrated that the deflection is consequently bounded 
near this critical load speed. Hosking, Sneyd, \& Waugh (1988) and Wang, Hosking \& Milinazzo (2004) showed that incorporating viscoelasticity in the linear theory via a memory function, corresponding to anelasticity consistent with the so-called standard model described in Squire, Hosking, Kerr \& Langhorne (1988) also predicted a large but finite response at $V=c_{\text {min }}$ together with some additional observed features (including the time lag). Thus while it emerged that a bounded response is always generated by a moving load, the development of a nonlinear viscoelastic theory was called for to more comprehensively treat the large amplitude responses near the critical speed $c_{m i n}$ in Wang, Hosking \& Milinazzo (2004).

The aim of the present contribution is the development of a versatile wave model which will allow the simulation of the wave response to a moving load under a wide range of conditions. In particular, we allow for two-dimensional waves created by a twodimensional load of arbitrary shape and weight distribution, and moving at an arbitrary time-dependent speed in the geometric configuration indicated in Figures 1 and 2. Our analysis incorporates nonlinearity, damping and non-zero thickness of the ice cover while retaining accurate treatment of all linear frequencies.

Our work is essentially motivated by an idea due to Whitham (1967), who proposed weakly nonlinear models in combination with the full dispersion of the water-wave problem. In the current context, the simplest such model would be the fully dispersive equation

$$
\eta_{t}+\frac{3}{2} \sqrt{g / H} \eta \eta_{x}+\frac{1}{2 \pi} \int_{-\infty}^{\infty} c(\xi) \hat{\eta}(\xi, t) e^{i \xi x} d \xi=0
$$

where the linear part of the equation is defined with the help of the dispersion relation $c(\xi)$. This relation which describes the phase speed $c$ of a linear periodic wave as a function of the wave number $\xi$, is given here by

$$
c^{2}(\xi)=\frac{g / \xi+\mathcal{D} \xi^{3} / \rho}{\operatorname{coth} \xi H+h \xi \rho_{I} / \rho} .
$$

This relation is defined in terms of the thickness of the elastic cover $h$, the fluid density $\rho$, the density of the elastic cover $\rho_{I}$, and the flexural rigidity of the elastic material $\mathcal{D}$ in addition to the undisturbed depth $H$ and gravitational acceleration $g$ defined above. The values of these parameters are tabulated for four experimental sites in Table 1 . The unknown $\eta(x, t)$ in the equation (1.1) is the deflection of the ice cover at a point $x$ and a time $t$, and $\hat{\eta}(\xi, t)$ is the Fourier transform given by

$$
\hat{\eta}(\xi, t)=\mathcal{F}\{\eta(\tilde{x}, t)\}(\xi)=\int_{-\infty}^{\infty} \eta(\tilde{x}, t) e^{-i \xi \tilde{x}} \mathrm{~d} \tilde{x} .
$$

Solutions of equation (1.1) describe only right-going waves, and there is no forcing or damping. In the present paper, in order to allow for the most possible flexibility we will derive a multi-directional system whose linear part corresponds to the full dispersion relation (1.2). In fact, by incorporating damping and rotatory inertia in the elastic description of the ice sheet, we obtain an even more general form of the dispersion relation (see (4.13)) which forms the basis for the linear part of our model.

A few nonlinear time-dependent models of flexural-gravity waves have appeared in the literature. In particular, long-wave equations of Boussinesq and KdV type have been proposed for general settings by Guyenne \& Părău (2012, 2014a); Marchenko (1988), and in particular for the study of waves in ice sheets on frozen rivers by Xia \& Shen (2002). One potential problem with long-wave equations is that they may model the flexural part of the dispersion relation inaccurately. Figures 3 and 4 show the dispersion relation for 


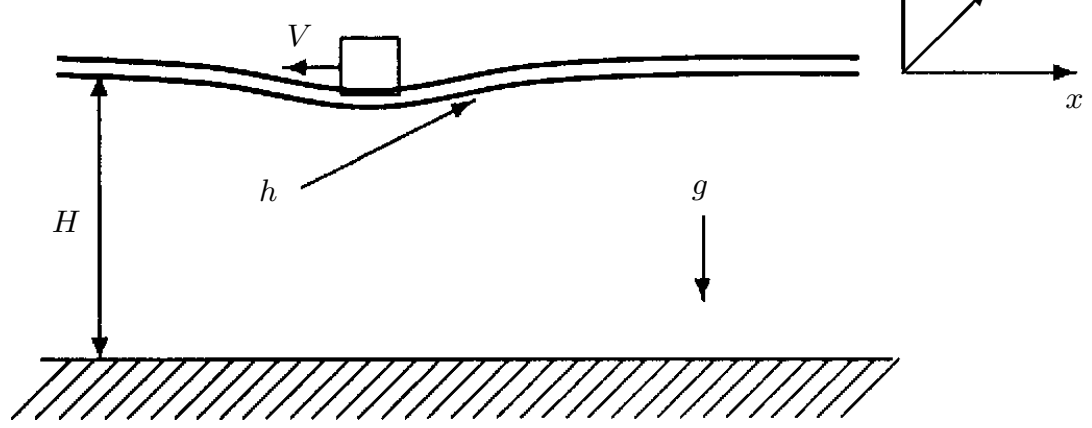

Figure 1. Geometry of the problem. $H$ is the fluid depth, $h$ is the thickness of the elastic layer, $g$ is gravity, $V$ is the velocity of the load.

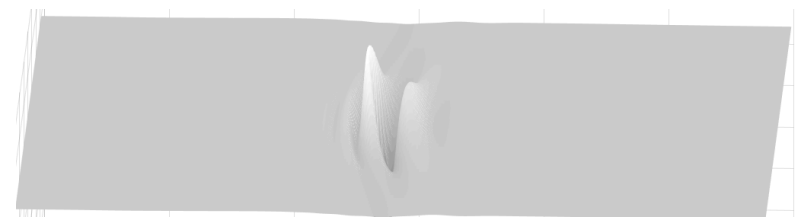

FiguRE 2. Two-dimensional wave response to a moving point load.

two cases under study in this paper. In the case shown in the left panel in Figure 3, the flexural rigidity of the the ice sheet is comparatively small, and long-wave models do not give an accurate description of the true linear dispersion relation.

Nonlinear models in the spirit of the nonlinear Schrödinger equation were also put forward for the study of flexural waves on relatively deep fluids. One interesting feature of these narrow-banded spectrum models is that they can be shown to exhibit modulational instability, as shown for example in Liu \& Mollo-Christensen (1988) and Marchenko (2016). Finally, fully nonlinear models such as proposed by Bonnefoy, Meylan \& Ferrant (2009) can also be used, but are more expensive with regard to computational time. The weakly nonlinear approximation coupled with the full dispersion relation as put forward in the present work allows significant savings in terms of computational time when compared to solving the full Euler equations for the underlying fluid flow problem such as used in Bonnefoy, Meylan \& Ferrant (2009); Guyenne \& Părău (2014b).

The disposition of the present paper is as follows. In the next section, the hydroelastic system is derived from the Kirchhoff-Love plate theory and the inviscid potential theory of surface waves. In Section 3, the dispersion relation is analyzed, and in section 4 , it is explained how this system is reduced to a weakly nonlinear formulation which nevertheless retains the full dispersion relation in the linear part. In section 5, the equations for two-dimensional wave patterns are presented, and in section 6, exact solutions of the linearized equations are found. These can be used to validate both the two-dimensional and three-dimensional codes, and to approximate some of the experimental data outright. Section 7 details the numerical method used, and Section 8 contains a number of numerical experiments including comparison with data from field campaigns and a study of decelerating loads. Our findings are summarized in Section 9. 


\begin{tabular}{|c|c|c|c|c|c|c|}
\hline Parameter & Symbol & Units & Lake Saroma & Cold Lake & Mille Lacs & McMurdo Sound \\
\hline Gravity & $g$ & $\mathrm{~m} \mathrm{~s}^{-2}$ & 9.81 & 9.81 & 9.81 & 9.81 \\
\hline Water density & $\rho$ & $\mathrm{kg} \mathrm{m}^{-3}$ & 1026 & 1024 & 1024 & 1024 \\
\hline Ice density & $\rho_{I}$ & $\mathrm{~kg} \mathrm{~m}^{-3}$ & 917 & 917 & 917 & 917 \\
\hline Water depth & $H$ & $\mathrm{~m}$ & 6.8 & 4.3 & 3.26 & 350 \\
\hline Ice thickness & $h$ & $\mathrm{~m}$ & 0.17 & 0.59 & 0.61 & 2.5 \\
\hline Elastic modulus & $E$ & $\mathrm{~N} \mathrm{~m}^{-2}$ & $5.1 \times 10^{8}$ & $4.9 \times 10^{9}$ & $9 \times 10^{9}$ & $5 \times 10^{9}$ \\
\hline Flexural rigidity & $\mathcal{D}$ & $\mathrm{Nm}$ & $2.35 \times 10^{5}$ & $9.4 \times 10^{7}$ & $1.91 \times 10^{8}$ & $7.32 \times 10^{9}$ \\
\hline Poisson ratio & $\nu$ & & 0.33 & 0.33 & 0.33 & 0.33 \\
\hline
\end{tabular}

Finally, Appendix A contains a comparison of steady wave profiles of a weakly nonlinear model to solutions of the hydro-elastic full Euler equations.

\section{The hydro-elastic system}

We consider irrotational motion of an inviscid and incompressible fluid of undisturbed mean depth $H$, and with gravity $g$ acting in the negative $z$-direction. The fluid is covered by an elastic solid layer which is described by the Kirchhoff-Love plate theory (cf. Squire, Hosking, Kerr \& Langhorne (1988)). For the sake of readability, we first treat the twodimensional problem, and return to the three-dimensional setting in Section 5 . The flow of the underlying liquid is described by the velocity potential $\phi(x, z, t)$ and by the fluid surface elevation $\eta(x, t)$ that coincides with the vertical deformation of the underside of the elastic cover. The fluid domain is the set $\left\{(x, z) \in \mathbb{R}^{2} \mid-H<z<\eta(x, t)\right\}$ extending to infinity in the positive and negative horizontal $x$ direction. The level $z=0$ corresponds to the fluid-solid interface at rest.

As explained for example in Whitham (1974), the fluid flow is governed by the Euler system consisting of the Laplace equation

$$
\phi_{x x}+\phi_{z z}=0 \quad \text { for } \quad x \in \mathbb{R}, \quad-H<z<\eta(x, t),
$$

the Neumann boundary condition at the flat bottom

$$
\phi_{z}=0 \quad \text { at } \quad z=-H
$$

the kinematic condition at the interface between the cover and the liquid

$$
\eta_{t}+\phi_{x} \eta_{x}-\phi_{z}=0 \quad \text { for } \quad x \in \mathbb{R}, \quad z=\eta(x, t),
$$

and the Bernoulli equation

$$
\phi_{t}+\frac{1}{2}|\nabla \phi|^{2}+g \eta+\frac{p}{\rho}=C_{B} \quad \text { for } \quad x \in \mathbb{R}, \quad z=\eta(x, t) .
$$

This constant $C_{B}$ will be specified below. As is common in hydro-elastic problems, we combine nonlinear equations for the fluid motion with linear elastic equations for the solid. This choice can be justified by noticing that liquid motions are of a different order of magnitude than deformations of the elastic solid cover. The presence of the overlying elastic solid is indicated via the pressure $p$ at the interface between the liquid and solid. This pressure is obtained from the beam equation for elastic solids. This equation is 
written as

$$
\mathcal{D} \partial_{x}^{4} \eta-\frac{\rho_{I} h^{3}}{12} \partial_{t}^{2} \partial_{x}^{2} \eta+\rho_{I} h \partial_{t}^{2} \eta+\rho_{I} g h+P-p=0 .
$$

This is a well known equation describing deflection $\eta(x, t)$ of beams. The second term in the equation which is due to horizontal acceleration of media particles is usually neglected, but in the present analysis, this term will actually allow an improved handling of the pressure imposed by a point load.

As already indicated in the introduction, it is important to include the effect of dissipation into the model. For the anelastic ice response to a moving load, the standard model of viscoelasticity (visualized as a spring in series with a Voigt unit) is considered to be most appropriate (see Hosking, Sneyd, \& Waugh (1988); Squire, Hosking, Kerr \& Langhorne (1988); Wang, Hosking \& Milinazzo (2004)). However, the simpler approach previously used for beams and adopted here assumes a damping force proportional to the vertical velocity, which results in the addition of a damping term $-b / h \partial_{t} \eta$ to the beam equation. The corresponding proportionality factor $b>0$ is assumed to be constant, and needs to be tuned for any given situation. The resulting equation is the beam equation in presence of damping which is given by

$$
\mathcal{D} \partial_{x}^{4} u_{2}-\frac{\rho_{I} h^{3}}{12} \partial_{t}^{2} \partial_{x}^{2} u_{2}+\rho_{I} h \partial_{t}^{2} u_{2}+b \partial_{t} u_{2}+\rho_{I} g h+P-p=0 .
$$

At this point one may wonder whether an improvement may be made by using more advanced beam models such as the Timoshenko theory which takes account of rotational bending effects, and is usually considered more precise (see Squire, Hosking, Kerr \& Langhorne (1988) for example). However, the use of such models is not very common, and it would also make the weakly nonlinear approximation explained in the next section much more difficult. Moreover, the Timoshenko theory's main advantage lies in the study of dynamics of either short beams or beams subjected to high-frequency excitation when the wavelength is near the media thickness, and neither of these are important in the situation at hand.

Assuming that the fluid foundation is always in contact with underside of the elastic plate (i.e. that there is no cavitation), and choosing the Bernoulli constant $C_{B}=\rho_{I} g h / \rho$ the beam equation (2.5) can be combined with the Bernoulli equation (2.4) by eliminating the pressure $p$ at the interface. The resulting equation be written in terms of the hydroelastic parameter $\varkappa=\mathcal{D} /(\rho g)$ in the form

$$
\varkappa g \partial_{x}^{4} \eta-\frac{\rho_{I} h^{3}}{12 \rho} \partial_{t}^{2} \partial_{x}^{2} \eta+\frac{\rho_{I} h}{\rho} \partial_{t}^{2} \eta+\frac{b}{\rho} \partial_{t} \eta+g \eta+\phi_{t}+\frac{1}{2}|\nabla \phi|^{2}+\frac{P}{\rho}=0 .
$$

This equation holds on the interface $z=\eta(x, t)$. Note that both the horizontal acceleration of the solid media particles and the nonlinear hydrodynamical effects are taken into account here. The load $P$ is considered to be a distributed pressure

$$
P(x, t)=\rho f\left(x-x_{0}-X(t)\right)
$$

moving along the $x$-axis at a velocity $X^{\prime}(t)$.

The main hydro-elastic system to be solved consists of the equation (2.1), together with the boundary conditions (2.3) and (2.6) with the exterior pressure (2.7). Note that the imposition of a point load will lead to usage of a Dirac delta function in Definition (2.7). This formal approach can be made mathematically precise in a number of ways (see Davys, Hosking \& Sneyd (1985) and Nevel (1970) for the steady case). In the present context, a rigorous formulation of an inhomogeneous problem with a point load will be given in the next section in the framework of the weakly nonlinear approximation. It 
also deserves notice that some authors advocate for taking account of the vertical inertia of the moving load mass in (2.7). However, this change would complicate the following analysis considerably. On the other hand, Squire, Hosking, Kerr \& Langhorne (1988) note that rotary inertia (the second term in (2.6)) must be included only if the loading is applied suddenly, or if it is of high frequency. As will come to light in Section 4, keeping the second term in (2.6) may actually be advantageous since it permits the treatment of a point load (2.7) in a mathematically consistent way.

\section{The dispersion relation}

In the situation depicted in Figure 1, but without load forcing, small-amplitude waves of the form $a \cos (\xi x-\xi c t)$ exist if the wave number $\xi$ and the the phase speed $c$ satisfy the dispersion relation (1.2). In stating this relation, the tacit assumption is made that the wavelength $\lambda=2 \pi / \xi$ is greater than the thickness of the elastic layer $h$. This assumption is generally reasonable, but for the sake of completeness, a more general dispersion relation is stated in (4.13). On the other hand, if the wavelength is very much longer than $h$, the term $h \xi$ is negligible, so that the dispersion relation (1.2) may be approximated by

$$
c^{2}=\frac{g}{\xi}\left[1+\xi^{4} \mathcal{D} / g \rho\right] \tanh \xi H .
$$

This approximate dispersion relation is used in Takizawa (1987) and many other works. Figures 3 and 4 show the two dispersion relations (1.2) and (3.1) for a number of parameter sets corresponding to different field experiments. Both dispersion relations have the same minimum wave speed $c_{\min }$ (up to a very small error). These critical wave speeds and the corresponding critical wavelengths are recorded in the captions of Figures 3 and 4 . The wave speed $c_{\text {min }}$ is singular in the sense that linear elastic theory predicts that the response to a point or distributed two-dimensional rectangular or circular load moving at this speed is unbounded (see Milinazzo, Shinbrot \& Evans (1995); Nugroho, Wang, Hosking \& Milinazzo (1999)). Thus $c_{\min }$ is a critical load speed in the traditional sense. A three-dimensional analysis shows that the load speed $\sqrt{g H}$ is not a critical speed, but it does mark the low load speed bound for the shadow zone predicted in Davys, Hosking \& Sneyd (1985). For loads moving below $c_{m i n}$, no wave response is observed (see section 8), and this is the quasi-static range according to Takizawa (1987).

Various other approximate dispersion relations have been used in the literature. In Xia \& Shen (2002), an approximation taking into account transversal loading and background shear was used, such as would be more common in the situation of an ice cover in a river. In the case of zero transversal loading and no background shear the dispersion relation reduces to

$$
c^{2}=\frac{g+\mathcal{D} \xi^{4} / \rho}{1 / H+h \xi^{2} \rho_{I} / \rho} .
$$

The purpose of the work of Xia \& Shen (2002) was the study of waves of ice sheets with axial loading, such as in frozen rivers. As a consequence, the equations are more similar to the shallow-water equations for river flow, and the authors advocate for the inclusion of nonlinear effects since the dispersion curve is such that many linear modes are in near resonance, i.e. with nearly the same linear phase speed. Since the focus of Xia \& Shen (2002) was not on moving loads, this approximation is not given further consideration.

A Boussinesq-type nonlinear system was found in Guyenne \& Părău (2014a), where 

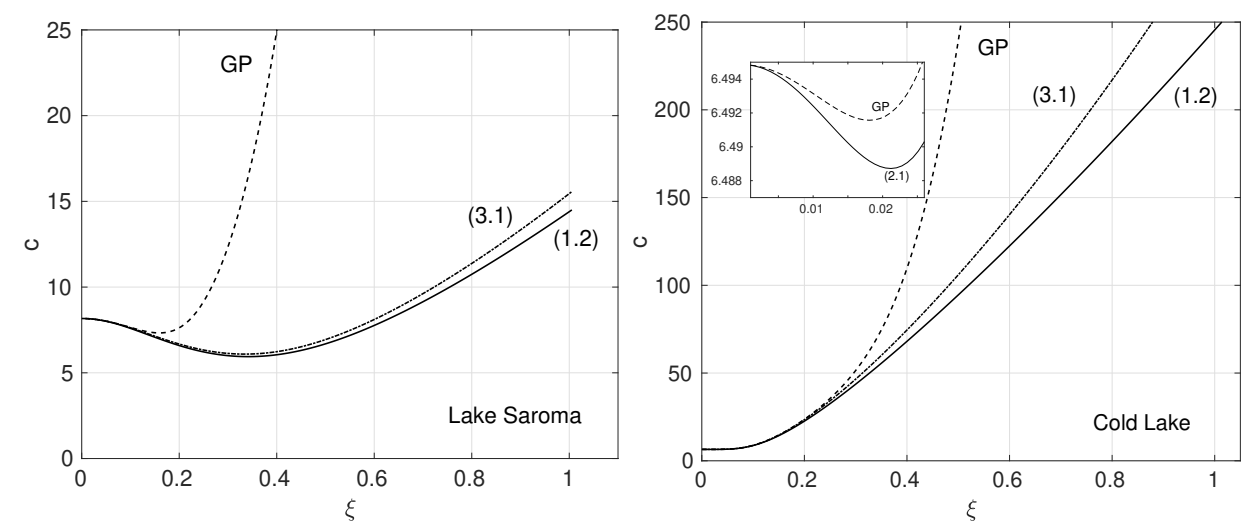

Figure 3. Dispersion curves for various approximations of the full dispersion relation. The phase speed $c$ is plotted as a function of wavenumber $\xi=2 \pi / \lambda$. The solid curves represent the full dispersion relation (1.2). The dashed-dotted curves represent the approximation (3.1). The dashed curve (GP) represents the dispersion relation from the Boussinesq model put forward by Guyenne \& Părău (2014a). The left panel shows the parameter values measured at Lake Saroma by Takizawa (1987). The minimum phase speed using(1.2) is $c_{\min }=5.94 \mathrm{~m} / \mathrm{s}$, and the corresponding wavelength is $\lambda_{\min }=18.6 \mathrm{~m}$. The right panel shows the parameter values measured at Cold Lake by Beltaos (1981) (see table 1). The minimum phase speed using (1.2) is $c_{\min }=6.49 \mathrm{~m} / \mathrm{s}$, and the corresponding wavelength is $\lambda_{\min }=296.3 \mathrm{~m}$.
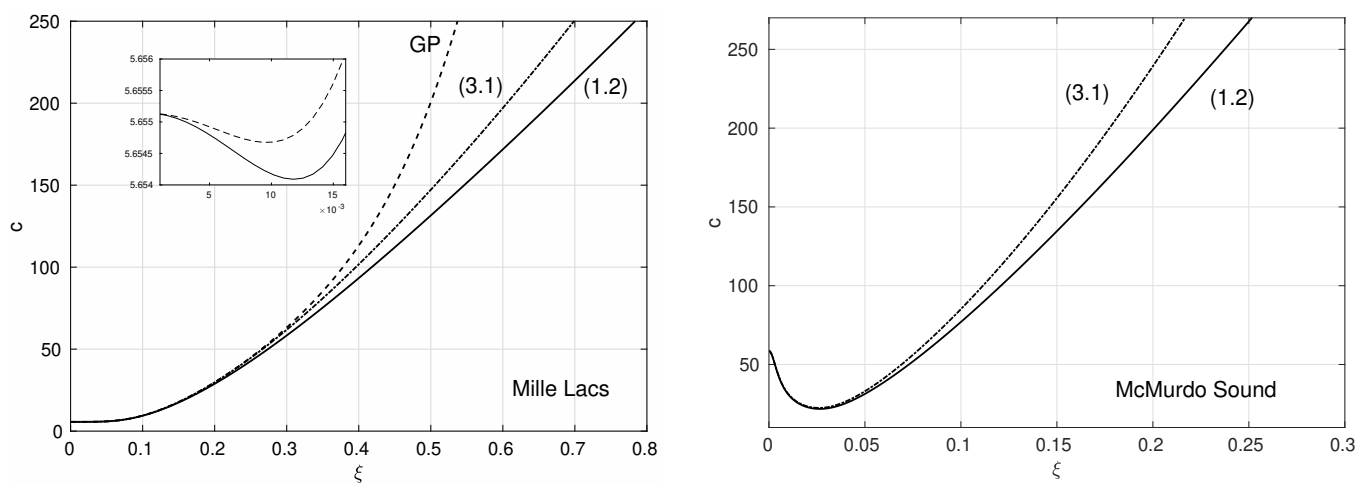

FiguRE 4. Dispersion curves for various approximations of the full dispersion relation. The phase speed $c$ is plotted as a function of wavenumber $\xi=2 \pi / \lambda$. The solid curves represent the full dispersion relation (1.2). The dashed-dotted curves represent the approximation (3.1). The dashed curve (GP) represents the dispersion relation from the Boussinesq model put forward by Guyenne \& Părău (2014a) (this curve is not shown in the right panel where the depth is very large). The left panel shows the parameter values measured at Mille Lacs (see Wilson (1955)). The minimum phase speed using $(1.2)$ is $c_{\min }=5.65 \mathrm{~m} / \mathrm{s}$, and the corresponding wavelength is $\lambda_{\text {min }}=533 \mathrm{~m}$. The right panel shows the parameter values measured at McMurdo Sound used by Davys, Hosking \& Sneyd (1985) (see table 1). The minimum phase speed using (1.2) is $c_{m i n}=21.8 \mathrm{~m} / \mathrm{s}$, and the corresponding wavelength is $\lambda_{m i n}=237 \mathrm{~m}$.

the approximate dispersion relation

$$
c^{2}=\left(g / \xi+D \xi^{3} / \rho\right)\left(H \xi-(H \xi)^{3} / 3+2(H \xi)^{5} / 15\right)
$$

appears if the system is linearized. As can be seen if Figures 3 and 4, the critical wave speed in the dispersion relation 3.3 differs from the critical wave speed provided by the full dispersion relation. The value of the critical speed is a matter of great practical 
importance for mechanized operations in cold regions, and it is therefore desirable to utilized a mathematical model which describes the critical speed as accurately as possible. In the next section, a more general approach is taken, and the full hydro-elastic system is approximated by a weakly nonlinear system.

\section{Weakly nonlinear approximation}

The main aim of this section is to find an approximation of the system (2.1)-(2.3), (2.6) in the weakly nonlinear framework. The system will also include a time and space dependent pressure forcing (2.7) in order to simulate a moving load on the ice sheet.

Considering the deflection of the ice cover $\eta(x, t)$ as above, and assuming irrotational flow in the fluid under the ice sheet, we introduce the surface trace of the velocity potential $\Phi(x, t)=\phi(x, \eta(x, t), t)$. Then the variable $u=\Phi_{x}=\phi_{x}+\eta_{x} \phi_{z}=\phi_{\tau} \sqrt{1+\eta_{x}^{2}}$, where $\phi_{\tau}$ is the fluid velocity tangent to the surface. It should be noted that due to the assumption of irrotational flow, and the resultant existence of the velocity potential, the unknowns $\eta$ and $\Phi$ can be used to describe the complete fluid motion as well as the deflection of the ice sheet.

We define the Dirichlet-Neumann operator $G(\eta)$ associated with the problem $(2.1)$, (2.2) and $\phi=\Phi$ on the domain bounded by the horizontal bottom and the curve $z=\eta$ by the formula

$$
G(\eta) \Phi=\left(\partial_{z} \phi-\partial_{x} \eta \partial_{x} \phi\right)_{z=\eta(x)}
$$

The dependence of the Dirichlet-Neumann operator $G$ on the deflection $\eta$ is nonlinear, but it is analytic in the sense explained in Nicholls \& Reitich (2001) and can be expanded as a power series as

$$
G(\eta) \Phi=\sum_{j=0}^{\infty} G_{j}(\eta) \Phi
$$

where each operator $G_{j}(\eta)$ is homogeneous of degree $j$ in powers of $\eta$. There is a well known recursion formula for the $G_{j}(\eta)$, and following Craig \& Groves (1994); Craig \& Sulem (1993), the first three terms have the form

$$
\begin{aligned}
& G_{0}(\eta)=D \tanh (H D), \quad G_{1}(\eta)=D \eta D-G_{0} \eta G_{0} \\
& G_{2}(\eta)=-\frac{1}{2}\left(|D|^{2} \eta^{2} G_{0}+G_{0} \eta^{2}|D|^{2}-2 G_{0} \eta G_{0} \eta G_{0}\right)
\end{aligned}
$$

where $D=-i \partial_{x}$ is the operator given by multiplication with $\xi$ in wavenumber space.

Using Equation (2.3) and Definition (4.1) leads to the equation

$$
\eta_{t}=G \Phi
$$

Note that this equation describes the kinematic boundary condition exactly. The second equation which is needed to describe the flow is found as follows. We first express derivatives of $\phi$ in terms of derivatives of $\eta$ and $\Phi$. The gradient $\nabla \phi$ is found from the definitions of $\Phi$ and $G$ in the form

$$
\nabla \phi=\left(\begin{array}{cc}
1 & \eta_{x} \\
-\eta_{x} & 1
\end{array}\right)^{-1}\left(\begin{array}{c}
\Phi_{x} \\
G \Phi
\end{array}\right)=\frac{1}{1+\eta_{x}^{2}}\left(\begin{array}{c}
\Phi_{x}-\eta_{x} G \Phi \\
G \Phi+\eta_{x} \Phi_{x}
\end{array}\right)
$$

Now differentiation of the surface potential $\Phi$ with respect to $t$ and applying (4.3) results in

$$
\Phi_{t}=\phi_{t}+\phi_{z} \eta_{t}=\phi_{t}+\phi_{z} G \Phi .
$$


This equation together with the velocity $\nabla \phi$ from (4.4) yields the acceleration potential of the inviscid fluid on surface in the form

$$
\phi_{t}+\frac{1}{2}|\nabla \phi|^{2}=\Phi_{t}+\frac{1}{2\left(1+\eta_{x}^{2}\right)}\left(\Phi_{x}^{2}-(G \Phi)^{2}\right)-\frac{1}{1+\eta_{x}^{2}} \eta_{x} \Phi_{x} G \Phi .
$$

Substituting Expression (4.5) into the Bernoulli equation (2.6) gives us the second governing equation for the unknowns $\eta$ and $\Phi$. Thus by means of the the DirichletNeumann operator $G(\eta)$ we transformed the two-dimensional problem (2.1)-(2.3), (2.6) to the one dimensional problem (4.3), (2.6) with (4.5). Note again that so far all manipulations have been formally exact, and no approximations have been introduced.

In order to approximate the one-dimensional problem above in the case of small surface deflections, the idea is to use a weakly nonlinear, but linearly fully dispersive system of evolution equations. In effect, in the equations (4.3) and (2.6) we discard all terms of cubic or higher order, and also all nonlinear dispersive terms. This procedure was justified in Moldabayev, Kalisch \& Dutykh (2015) by using an exponential scaling, but it can also be viewed as simply keeping all linear error terms as they do not change the order of the approximation. The equation is thus formally of the same order as the corresponding Boussinesq equation, but including the exact form of the linear terms gives a decisive advantage when it comes to describing flexural-gravity waves. The idea of keeping various corrections of a lower order has been used in a number of other cases, especially in the context of coastal engineering (see for example Madsen, Murray \& Sørensen (1991); Nwogu (1993); Wei, Kirby, Grilli \& Subramanya (1995)). Thus using this idea, the first term in the series (4.2) $G_{0}=D \tanh H D$ is kept unchanged, while the term $G_{1}$ is simplified to $G_{1}(\eta)=D \eta D=-\partial_{x} \eta \partial_{x}$. This approximation immediately transforms (4.3) to the simplified equation

$$
\eta_{t}=G_{0} \Phi-\partial_{x}\left(\eta \Phi_{x}\right) .
$$

Next, the equation (2.6) is simplified. First, we aim to remove the second time derivatives by means of applying (4.3) iteratively. Anticipating that cubic and higher-order nonlinearities will not be carried through, we approximate the Dirichlet-Neumann operator by $G=G_{0}+G_{1}+G_{2}$. We will temporarily use the notation

$$
G_{2}\left(\eta_{1}, \eta_{2}\right)=-\frac{1}{2}\left(|D|^{2} \eta_{1} \eta_{2} G_{0}+G_{0} \eta_{1} \eta_{2}|D|^{2}-2 G_{0} \eta_{1} G_{0} \eta_{2} G_{0}\right)
$$

which reduces to our regular notation $G_{2}(\eta)=G_{2}(\eta, \eta)$ in the case when $\eta_{1}=\eta_{2}$. Then taking into account that time and spatial derivatives commute, it can be seen that

$$
\begin{aligned}
\partial_{t} G_{2}(\eta) \Phi= & G_{2}\left(\eta_{t}, \eta\right) \Phi+G_{2}\left(\eta, \eta_{t}\right) \Phi+G_{2}(\eta, \eta) \Phi_{t} \\
& =G_{2}\left(\left(G_{0}+G_{1}+G_{2}\right) \Phi, \eta\right) \Phi+G_{2}\left(\eta,\left(G_{0}+G_{1}+G_{2}\right) \Phi\right) \Phi+G_{2}(\eta, \eta) \Phi_{t} .
\end{aligned}
$$

Treating $\partial_{t} G_{1}(\eta) \Phi$ in a similar manner, applying (4.3) again and truncating nonlinearity at the third order we eventually arrive at the relation

$$
\partial_{t}^{2} \eta=\left(G_{0}+G_{1}(\eta)+G_{2}(\eta)\right) \Phi_{t}+G_{1}\left(G_{0} \Phi\right) \Phi .
$$

This identity together with (4.3) and (4.5) is substituted into (2.6), so that we find

$$
F \Phi_{t}=-g\left(1+\varkappa \partial_{x}^{4}\right) \eta-\frac{b}{\rho}\left(G_{0}+G_{1}(\eta)\right) \Phi-\frac{\rho_{I} h}{\rho}\left(1-\frac{h^{2} \partial_{x}^{2}}{12}\right) G_{1}\left(G_{0} \Phi\right) \Phi-\frac{1}{2} \Phi_{x}^{2}-\frac{P}{\rho},
$$

where the operator $F$ is defined by

$$
F=K+\frac{\rho_{I} h}{\rho}\left(1-\frac{h^{2} \partial_{x}^{2}}{12}\right)\left(G_{1}(\eta)+G_{2}(\eta)\right)
$$


and the operator $K$ is defined by

$$
K=1+\frac{\rho_{I} h}{\rho}\left(1-\frac{h^{2} \partial_{x}^{2}}{12}\right) G_{0} .
$$

In wavenumber space, $K$ has the expression

$$
k(\xi)=1+\frac{\rho_{I} h}{\rho} \xi \tanh H \xi+\frac{\rho_{I}}{\rho} \frac{h^{3}}{12} \xi^{3} \tanh H \xi,
$$

which shows that $K$ can easily be inverted. Thus equation (4.7) can be simplified further by taking inverse of the operator $F$ as follows

$$
F^{-1}=K^{-1}-\frac{\rho_{I} h}{\rho}\left(1-\frac{h^{2} \partial_{x}^{2}}{12}\right) K^{-1}\left(G_{1}(\eta)+G_{2}(\eta)\right) K^{-1}+\left(\frac{\rho_{I} h}{\rho}\right)^{2} K^{-1} G_{1}(\eta) K^{-1} G_{1}(\eta) K^{-1}
$$

where higher-order terms in $\eta$ have been omitted. We introduce the notation

$$
\Gamma=\frac{1}{\rho} F^{-1} P
$$

where the inverse is understood in the sense of (4.10), and the function $\Gamma(x, t)$ depends on both the surface elevation $\eta(x, t)$ and the imposed pressure $P(x, t)$. Thus applying (4.10) to both sides of the expression (4.7), discarding highly nonlinear and nonlinear dispersive terms and simplifying yields the equation

$$
\Phi_{t}=-g \frac{1+\varkappa D^{4}}{K} \eta-\frac{b}{\rho} \frac{G_{0}}{K} \Phi-\frac{\rho_{I} g h}{2 \rho} \partial_{x}^{2} \eta^{2}+\frac{b}{\rho} \partial_{x}\left(\eta \Phi_{x}\right)-\frac{1}{2} \Phi_{x}^{2}-\Gamma .
$$

The equations (4.6), (4.12) give a one-dimensional fully dispersive weakly nonlinear approximation of the problem (2.1)-(2.3), (2.6).

Looking for solutions of the homogeneous linearization in the form $\eta(x, t)=A e^{i \xi x-i \omega t}$, $\Phi(x, t)=B e^{i \xi x-i \omega t}$ gives rise to the necessary condition

$$
\omega^{2}+\frac{i b}{\rho} \frac{\xi \tanh H \xi}{k(\xi)} \omega-g\left(1+\varkappa \xi^{4}\right) \frac{\xi \tanh H \xi}{k(\xi)}=0 .
$$

In terms of the phase speed $c=\omega(\xi) / \xi$, the dispersion relation is written as

$$
c^{2}+\frac{i b}{\rho} \frac{\tanh H \xi}{k(\xi)} c-g\left(1+\varkappa \xi^{4}\right) \frac{\tanh H \xi}{\xi k(\xi)}=0 .
$$

So far all approximations have been made for a general inhomogeneity $P(x, t)$. Considering in particular a moving load (2.7) firstly one calculates

$$
w(x, t)=K^{-1} P / \rho=\frac{1}{2 \pi} \int_{\mathbb{R}} \frac{\widehat{f}(\xi) e^{i\left(x-x_{0}-X(t)\right) \xi}}{k(\xi)} d \xi
$$

where the operator $K$ and its symbol $k(\xi)$ are defined by (4.8) and (4.9). Note that since the rotatory inertia term, i.e. the third term in (4.9) is included in the definition of $k(\xi)$, this integral is convergent even in the case of a point load. In fact, if $f(x)=\gamma \delta(x)$ with $\widehat{f}(\xi) \equiv \gamma$, the function $w$ is well defined and bounded. The regularity of this function permits the omission of the quadratic parts with respect to $\eta$ in (4.11) since these parts are highly dispersive and nonlinear. Thus the presence of the load results in the forcing term

$$
\Gamma=w-\frac{\rho_{I} h}{\rho}\left(1-\frac{h^{2} \partial_{x}^{2}}{12}\right) K^{-1} G_{1}(\eta) w .
$$


Bi-directional weakly nonlinear systems such as (4.6), (4.12) have only appeared recently in the literature. Aceves-Sánchez, Minzoni \& Panayotaros (2013) and VargasMagana \& Panayotaros (2016) studied a Whitham-type system for free surface waves in the presence of non-trivial bottom topography. In Carter (2018) and Moldabayev, Kalisch \& Dutykh (2015) the fidelity of Whitham-type systems when compared with Euler flows and lab experiments is under review. One interesting feature of these models is that periodic solutions exist (see Ehrnström \& Kalisch (2009, 2013)), and that they feature modulational instability in a similar fashion as deep-water wave models (see Hur \& Johnson (2015a,b); Sanford, Kodama, Carter \& Kalisch (2014)). As far as we know, the only result pertaining to multi-directional fully dispersive systems is given by Lannes \& Saut (2013), where it is assumed that perturbations in the direction transverse to the main direction of wave propagation are weak. In the next section, we derive a multidirectional fully dispersive system without that assumption.

\section{2D weakly nonlinear approximation}

Regarding now the fluid surface displacement $\eta(x, y, t)$ as a function of two spatial variables we introduce the surface velocity potential $\Phi(x, y, t)=\phi(x, y, \eta(x, y, t), t)$. In this case, the first two terms of the Dirichlet-Neumann operator have the form

$$
G_{0}=|D| \tanh (H|D|), \quad G_{1}(\eta)=-\partial_{x} \eta \partial_{x}-\partial_{y} \eta \partial_{y}-G_{0} \eta G_{0}
$$

where $D=\left(-i \partial_{x},-i \partial_{y}\right)$ and $|D|=\sqrt{-\Delta}=\sqrt{-\partial_{x}^{2}-\partial_{y}^{2}}$. We define the operator $K$ by

$$
K=1+\frac{\rho_{I} h}{\rho}\left(1-\frac{h^{2} \Delta}{12}\right) G_{0}
$$

with the symbol

$$
k\left(\xi_{1}, \xi_{2}\right)=1+\frac{\rho_{I} h}{\rho}\left(1+\frac{h^{2}}{12}\left(\xi_{1}^{2}+\xi_{2}^{2}\right)\right) \sqrt{\xi_{1}^{2}+\xi_{2}^{2}} \tanh \left(H \sqrt{\xi_{1}^{2}+\xi_{2}^{2}}\right) .
$$

The formal derivation of the previous section can be used in the same way without any substantial changes. The final two-dimensional system to be solved is

$$
\begin{gathered}
\eta_{t}=G_{0} \Phi-\partial_{x}\left(\eta \Phi_{x}\right)-\partial_{y}\left(\eta \Phi_{y}\right), \\
\Phi_{t}=-g \frac{1+\varkappa \Delta^{2}}{K} \eta-\frac{b}{\rho} \frac{G_{0}}{K} \Phi-\Gamma-\frac{1}{2} \Phi_{x}^{2}-\frac{1}{2} \Phi_{y}^{2}-\frac{\rho_{I} g h}{2 \rho} \Delta \eta^{2}+\frac{b}{\rho} \partial_{x}\left(\eta \partial_{x} \Phi\right)+\frac{b}{\rho} \partial_{y}\left(\eta \partial_{y} \Phi\right),
\end{gathered}
$$

with

$$
\Gamma=w-\frac{\rho_{I} h}{\rho}\left(1-\frac{h^{2} \Delta}{12}\right) K^{-1} G_{1}(\eta) w
$$

and

$$
w(x, y, t)=K^{-1} P / \rho
$$

where the operator $K$ and the corresponding symbol $k(\xi)$ are defined by (5.1) and (5.2). In case of the distributed moving load

$$
P(x, y, t)=\rho f\left(x-x_{0}-X(t), y\right)
$$

one finds

$$
w(x, y, t)=\frac{1}{(2 \pi)^{2}} \int_{\mathbb{R}^{2}} \frac{e^{i\left(x-x_{0}-X(t)\right) \xi_{1}+i y \xi_{2}} \widehat{f}\left(\xi_{1}, \xi_{2}\right)}{k\left(\xi_{1}, \xi_{2}\right)} d \xi_{1} d \xi_{2}
$$


It is clear that also in this case a point load $f(x, y)=\delta(x, y)$ will lead to a smooth function $w$ with respect to spatial variables. This justifies making use of the system (5.3)-(5.4) even for the load concentrated at a point. It is worth notice that this feature is achieved by keeping rotary inertia which is usually neglected by other authors in (2.6). Thus there is no need for regularization of the point load as it is smoothed naturally in our framework by the inverse operator $K^{-1}$.

\section{Exact solution of the linearized problem}

It turns out that after further simplification of (5.3)-(5.4) the new system can be solved exactly. First we consider the linearized problem without an imposed pressure. This simplification leads to the homogeneous linear system

$$
\begin{gathered}
\eta_{t}=G_{0} \Phi \\
\Phi_{t}=-g \frac{1+\varkappa \Delta^{2}}{K} \eta-\frac{b}{\rho} \frac{G_{0}}{K} \Phi .
\end{gathered}
$$

Introducing the operators

$$
R=\frac{b G_{0}}{2 \rho K}
$$

and

$$
U=\sqrt{\frac{g\left(1+\varkappa D^{4}\right) G_{0}}{K}-R^{2}},
$$

one can easily solve the new system exactly. In terms of initial values $\eta_{0}, \Phi_{0}$, the solution is given by

$$
\begin{gathered}
\eta^{H L}(t)=e^{-R t}\left(\frac{R \sin (U t)}{U}+\cos (U t)\right) \eta_{0}+e^{-R t} \frac{G_{0} \sin (U t)}{U} \Phi_{0}, \\
\Phi^{H L}(t)=e^{-R t}\left(-g \frac{1+\varkappa \Delta^{2}}{K}\right) \frac{\sin (U t)}{U} \eta_{0}+e^{-R t}\left(\frac{-R \sin (U t)}{U}+\cos (U t)\right) \Phi_{0} .
\end{gathered}
$$

Notice that operator $R$ is associated with the viscosity of the ice, and these solutions are damped very quickly.

The next level of accuracy is to discard again all nonlinear terms in System (5.3)-(5.4) and use a slightly simplified expression for the imposed pressure $\Gamma$. It turns out that the second term in the expression for $\Gamma$ given in (5.5) does not affect the solution a great deal. Indeed one may omit the term depending on $G_{1}(\eta)$, and use the approximate form $\Gamma=w$. The numerical scheme was run both with and without this approximation, and there was no discernible difference in the solution. Thus the new two-dimensional linear system to be solved is

$$
\begin{gathered}
\eta_{t}=G_{0} \Phi \\
\Phi_{t}=-g \frac{1+\varkappa \Delta^{2}}{K} \eta-\frac{b}{\rho} \frac{G_{0}}{K} \Phi-w .
\end{gathered}
$$

For constant load speed $X^{\prime}(t)=V$, a closed-form solution of this system can be found using the Laplace transformation $\mathcal{L}$. Let $\widehat{\eta}, \widehat{\Phi}$ and $\widehat{w}$ be Laplace transforms of $\eta, \Phi$ and $w$, respectively. Then the system is transformed to the system

$$
\widehat{\eta}(s)=\frac{1}{s^{2}+2 R s+R^{2}+U^{2}}\left((s+2 R) \eta_{0}+G_{0} \Phi_{0}-G_{0} \widehat{w}(s)\right),
$$


where

$$
\widehat{\Phi}(s)=\frac{1}{s^{2}+2 R s+R^{2}+U^{2}}\left(-\left(R^{2}+U^{2}\right) G_{0}^{-1} \eta_{0}+s \Phi_{0}-s \widehat{w}(s)\right),
$$

$$
\widehat{w}(x, y, s)=\frac{1}{(2 \pi)^{2}} \int_{\mathbb{R}^{2}} \frac{e^{i\left(x-x_{0}\right) \xi_{1}+i y \xi_{2}} \widehat{f}\left(\xi_{1}, \xi_{2}\right)}{\left(s+i \xi_{1} V\right) k\left(\xi_{1}, \xi_{2}\right)} d \xi_{1} d \xi_{2} .
$$

The solution of this system has the form $\eta(t)=\eta^{H L}(t)+\eta^{w}(t)$ and $\Phi(t)=\Phi^{H L}(t)+\Phi^{w}(t)$, where

$$
\begin{aligned}
\eta^{w}(t) & =\mathcal{L}^{-1}\left(\frac{-G_{0}}{(s+R)^{2}+U^{2}} \widehat{w}(s)\right), \\
\Phi^{w}(t) & =\mathcal{L}^{-1}\left(\frac{-s}{(s+R)^{2}+U^{2}} \widehat{w}(s)\right) .
\end{aligned}
$$

And so we have

$\eta^{w}(x, y, t)=\mathcal{F}^{-1}\left(A_{\eta}(\xi) e^{-R(\xi) t-i U(\xi) t}+B_{\eta}(\xi) e^{-R(\xi) t+i U(\xi) t}+C_{\eta}(\xi) e^{-i \xi_{1} V t}\right)\left(x-x_{0}, y\right)$,

$\Phi^{w}(x, y, t)=\mathcal{F}^{-1}\left(A_{\Phi}(\xi) e^{-R(\xi) t-i U(\xi) t}+B_{\Phi}(\xi) e^{-R(\xi) t+i U(\xi) t}+C_{\Phi}(\xi) e^{-i \xi_{1} V t}\right)\left(x-x_{0}, y\right)$,

where the Fourier variable is $\xi=\left(\xi_{1}, \xi_{2}\right)$, and the functions $A_{\eta}, B_{\eta}$ and $C_{\eta}$, are defined by

$$
\begin{gathered}
A_{\eta}(\xi)=-\frac{\widehat{f}(\xi) k(\xi)^{-1} G_{0}(\xi)}{2 i U(\xi)\left(R(\xi)+i U(\xi)-i \xi_{1} V\right)}, \\
B_{\eta}(\xi)=\frac{\widehat{f}(\xi) k(\xi)^{-1} G_{0}(\xi)}{2 i U(\xi)\left(R(\xi)-i U(\xi)-i \xi_{1} V\right)}, \\
C_{\eta}(\xi)=-\frac{\widehat{f}(\xi) k(\xi)^{-1} G_{0}(\xi)}{\left(R(\xi)+i U(\xi)-i \xi_{1} V\right)\left(R(\xi)-i U(\xi)-i \xi_{1} V\right)},
\end{gathered}
$$

and correspondingly we have

$$
\begin{gathered}
A_{\Phi}(\xi)=\frac{\widehat{f}(\xi) k(\xi)^{-1}(R(\xi)+i U(\xi))}{2 i U(\xi)\left(R(\xi)+i U(\xi)-i \xi_{1} V\right)}, \\
B_{\Phi}(\xi)=-\frac{\widehat{f}(\xi) k(\xi)^{-1}(R(\xi)-i U(\xi))}{2 i U(\xi)\left(R(\xi)-i U(\xi)-i \xi_{1} V\right)}, \\
C_{\Phi}(\xi)=\frac{\widehat{f}(\xi) k(\xi)^{-1} i \xi_{1} V}{\left(R(\xi)+i U(\xi)-i \xi_{1} V\right)\left(R(\xi)-i U(\xi)-i \xi_{1} V\right)} .
\end{gathered}
$$

These formulae represent the exact solution of the linear system (6.5)-(6.6) in the case of constant load speed. These formulae may be used to check the numerical algorithm put forward in the next section, and they are also of independent interest as they can by implemented with relative ease.

\section{Numerical treatment of general nonlinear system}

An effective way of approximating the full system (5.3)-(5.4) numerically is to treat the linear and nonlinear parts separately using a split-step scheme. To be more specific, the system (5.3)-(5.4) is represented in the form

$$
\mathcal{Z}_{t}=\mathcal{A}(\mathcal{Z})+\mathcal{B}(\mathcal{Z}, t),
$$


and each time step is split into two parts where the systems $\mathcal{Z}_{t}=\mathcal{A}(\mathcal{Z})$ and $\mathcal{Z}_{t}=\mathcal{B}(\mathcal{Z}, t)$ are solved separately. Here the first differential equation corresponds to the homogeneous linear system (6.1)-(6.2). More precisely, the solution vector is $\mathcal{Z}=(\eta, \Phi)$ and the system has the form

$$
\left[\begin{array}{l}
\eta_{t} \\
\Phi_{t}
\end{array}\right]=\left[\begin{array}{l}
\mathcal{A}_{1}(\mathcal{Z}) \\
\mathcal{A}_{2}(\mathcal{Z})
\end{array}\right]
$$

where $\mathcal{A}_{1}(\mathcal{Z})=G_{0} \Phi$ and $\mathcal{A}_{2}(\mathcal{Z})=-g\left(1+\varkappa \Delta^{2}\right) K^{-1} \eta-b G_{0}(\rho K)^{-1} \Phi$. Its exact solution (6.3)-(6.4) represents the integrator $\exp (t \mathcal{A})$ of the first system. As for the second differential equation with $\mathcal{B}(\mathcal{Z}, t)$ one needs to be careful since in general numerical splitting schemes are developed only for autonomous equations. This is not the case with the second system $\ddagger_{t}=\mathcal{B}(\mathcal{Z}, t)$ having the form

$$
\left[\begin{array}{l}
\eta_{t} \\
\Phi_{t}
\end{array}\right]=\left[\begin{array}{c}
\mathcal{B}_{1}(\mathcal{Z}) \\
\mathcal{B}_{2}(\mathcal{Z}, t)
\end{array}\right]
$$

where

$$
\begin{gathered}
\mathcal{B}_{1}(\mathcal{Z})=-\partial_{x}\left(\eta \Phi_{x}\right)-\partial_{y}\left(\eta \Phi_{y}\right) \\
\mathcal{B}_{2}(\mathcal{Z}, t)=-\Gamma(\eta, t)-\frac{1}{2} \Phi_{x}^{2}-\frac{1}{2} \Phi_{y}^{2}-\frac{\rho_{I} g h}{2 \rho} \Delta \eta^{2}+\frac{b}{\rho} \partial_{x}\left(\eta \partial_{x} \Phi\right)+\frac{b}{\rho} \partial_{y}\left(\eta \partial_{y} \Phi\right) .
\end{gathered}
$$

This system contains the pressure forcing and all nonlinear terms. Note that the righthand side depends on time $t$.

Now let $\delta t$ denote a time step. Suppose we know the solution $z(t)$ of the equation $\mathcal{Z}_{t}=\mathcal{B}(\mathcal{Z}, t)$ associated with the system $\mathcal{Z}_{t}=\mathcal{B}(\mathcal{Z}, t)$ at time $t$. To find its solutions at time $t+\delta t$, we solve it making use of a standard numerical scheme, as for example the four-stage Runge-Kutta method. However, for use in the the split-step scheme it needs to be modified slightly in a semi-autonomous way as follows. If $\mathcal{Z}_{i}$ is a value at the beginning of a substep with the length $\delta t_{i}$ then the value $z \mathcal{Z}_{i+1}$ is defined by $\mathcal{Z}_{i+1}=\mathcal{Z}_{i}+\left(F_{1}+2 F_{2}+2 F_{3}+F_{4}\right) / 6$, where

$$
\begin{aligned}
& F_{1}=\delta t_{i} \mathcal{B}\left(\mathcal{Z}_{i}, t\right), \\
& F_{2}=\delta t_{i} \mathcal{B}\left(\mathcal{Z}_{i}+F_{1} / 2, t\right) \\
& F_{3}=\delta t_{i} \mathcal{B}\left(\mathcal{Z}_{i}+F_{2} / 2, t\right), \\
& F_{4}=\delta t_{i} \mathcal{B}\left(\mathcal{Z}_{i}+F_{3}, t\right) .
\end{aligned}
$$

Note that the time $t$ is fixed here. The procedure defines the numerical integrator $\exp \left(\delta t_{i} \mathcal{B}\right)$ on a substep of the time interval $(t, t+\delta t)$. The integrator $\exp (\delta t(\mathcal{A}+\mathcal{B}))$ of the whole system (7.1) is defined as an integrator of 6th-order which is thoroughly described in Dinvay, Dutykh \& Kalisch (2019), so that we do not go into any more detail here.

In order to solve each substep, we use a Fourier spectral discretization for the spatial part, where the nonlinear terms are evaluated with the fast Fourier transform.

\section{Numerical experiments}

In this section, we test our model on a number of cases provided by the experimental campaigns carried out on Mille Lacs, Minnesota, USA by Wilson (1955), at Cold Lake in Canada by Beltaos (1981), and on Lake Saroma in Japan by Takizawa (1987, 1988). A number of additional important field campaigns have been conducted over the years, most notably the work conducted at McMurdo sound in Antarctica and reported in Squire, Robinson, Langhorne \& Haskell (1988) and Squire, Hosking, Kerr \& Langhorne 

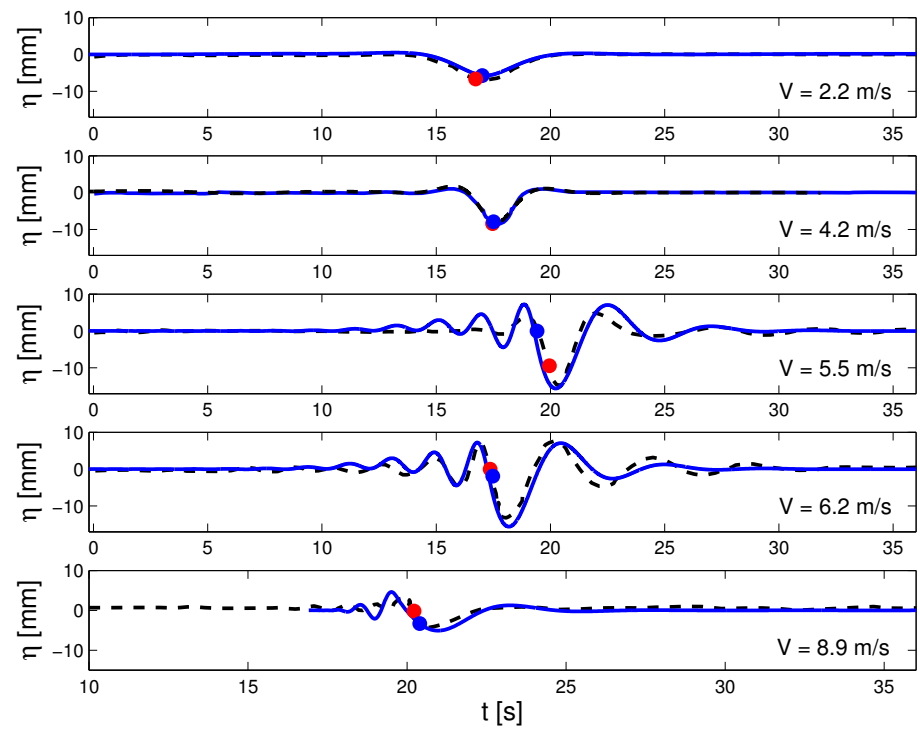

FiguRE 5. Comparison of numerical approximation of equations (4.6), (4.12) and experimental ice deflection records from the experiments of Takizawa (1987). The load speeds are $2.2 \mathrm{~m} / \mathrm{s}$, $4.2 \mathrm{~m} / \mathrm{s}, 5.5 \mathrm{~m} / \mathrm{s}, 6.2 \mathrm{~m} / \mathrm{s}$, and $8.9 \mathrm{~m} / \mathrm{s}$ (from top to bottom). The dashed black curves are the experimental data of Takizawa (1987), and the red dots indicate the $z$-position of the skidoo used in these experiments at the time it passes the deflectometer. The blue curves represent the a time series of the numerical approximation of equations (4.6), (4.12), taken at a measuring station corresponding to the position of the deflectometer in the field experiment. The blue dots represent the $z$-position of the load as it passes the $x$-position where the time series is obtained.
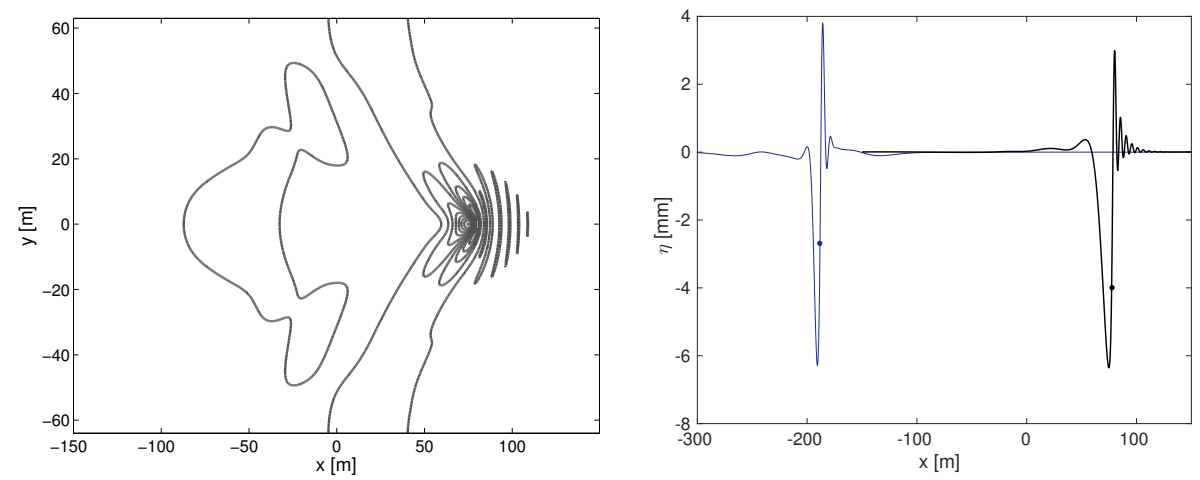

Figure 6. Numerical approximation of equations (5.3), (5.4) for constant load velocity $V=8.9 \mathrm{~m} / \mathrm{s}$, such as in Figure 5 . The left panel shows the wave crest pattern, and the right panel shows the deflection of the ice sheet along the center line $y=0$. For comparison, the solution of equations $(4.6),(4.12)$ is also plotted in the right panel. This graph is shifted left for easier comparison.

(1988). However, in this campaign, a strain gauge was instead of a deflectometer. Using a strain gauge has certain practical advantages, but makes a comparison to numerical simulations more difficult.

We first focus on experiments of a Japanese research group headed up by Takatoshi Takizawa. These experiments were conducted on Lake Saroma on the island of Hokkaido in Japan. The lake was covered with an icesheet of about $0.16 \mathrm{~m}$ thickness which had a 

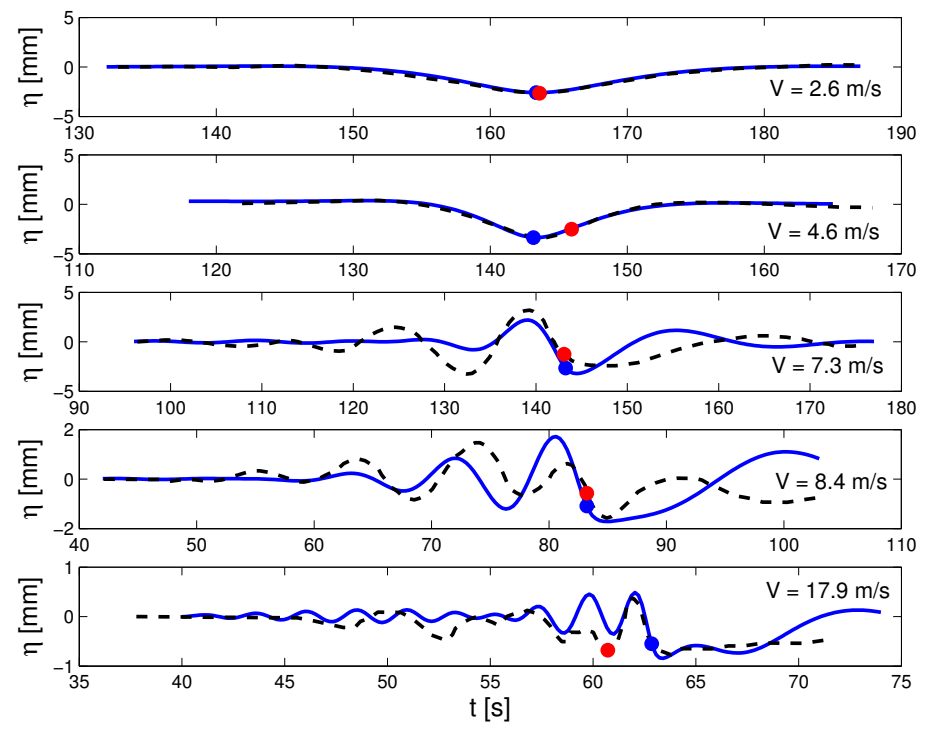

FiguRE 7. Comparison of numerical approximation of equations (5.3), (5.4) and experimental ice deflection records from the single truck experiments of Wilson (1955). The dashed black curves are the experimental data of Wilson (1955), and the red dots indicate the $z$-position of the truck at the time it passes the deflectometer. The blue curves represent the numerical approximation of equations (5.3), (5.4), and the blue dots represent the $z$-position of the load at the time is passes the deflectometer.

light snow cover of about 2 to $8 \mathrm{~cm}$. A skidoo weighing $235 \mathrm{~kg}$ was driven on a test track about $200 \mathrm{~m}$ long. Deflectometers and vehicle detectors were installed in several locations along the track. The flexural properties of the ice were determined with static loading tests, and the most important physical parameters in these experiments are summarized in Table 1.

In the following, our aim will be to compare numerical approximations of the system (4.6), (4.12) with results from Takizawa's experimental data. For this purpose, Figure 6 of Takizawa (1987) has been digitized. This figure shows typical deflectometer records from skidoo passages on February 5th, 1981. It can be seen in Figure 5 that the main features of the experimental data can be found in the numerical solutions. The quasi-static cases of load velocity $2.2 \mathrm{~m} / \mathrm{s}$ and $4.2 \mathrm{~m} / \mathrm{s}$ which are well below the minimum phase speed shown in the dispersion curve (left panel of Figure 3) are matched almost perfectly. The two-wave stage with a speed of $6.2 \mathrm{~m} / \mathrm{s}$ also shows good agreement between experiment and numerical simulation, and in particular, both the flexural and the gravity wave are captured in the computed solution. The single-wave stage at load velocity $8.9 \mathrm{~m} / \mathrm{s}$ which is above the limiting long-wave speed gives a fairly good fit, but the leading waves are slightly exaggerated. The case of load speed $5.5 \mathrm{~m} / \mathrm{s}$ which is close to the critical speed dividing the range of quasi-static and two-wave regimes features a few spurious leading waves in the computed solution. Nevertheless, even in this case, the difference between maximum and minimum deflection is captured fairly well. It should be noted that this computation was done using the one-dimensional model (4.6), (4.12). Running this case with a one-dimensional model necessitates adjustment of the load (as done for example also in Părău \& Dias (2002). The load was adjusted by aiming for an optimal fit in a static loading case, i.e. with zero load velocity.

It should also be emphasized that the same damping coefficient $b$ was used in all five 
of the computations shown in Figure 5. The damping is due to a number of factors such as inherent viscous damping, snow cover and damping in the turbulent boundary layer in the underlying fluid base. The coefficient $b$ is non-dimensionalized by setting $b=B b_{c}$, where $b_{c}$ is defined as $b_{c}=2 \sqrt{\rho g \rho_{I} h}$. The coefficient $B$ then needed to be determined by trial and error, but the process can be optimized by choosing $B$ in such a way that the time lag is optimized (see Fig. 11 in Takizawa (1987)). In the case of fitting the records of Takizawa, we determined $B=0.41$ for a best fit.

Previous attempts to match the experimental data from Takizawa (1987) were made by Takizawa himself in Takizawa (1987) who obtained good qualitative agreement, but did not aim for quantitative agreement. Fair quantitative agreement was obtained in Milinazzo, Shinbrot \& Evans (1995), but the numerical data needed to be symmetric for subcritical load speeds and did not feature temporal localization for super-critical load speeds due to the steady nature of their model. The present work removes both of these impairments.

We also made some comparisons with the two-dimensional model (5.3), (5.4), which gave similar results. An example of a two-dimensional wave pattern is shown in Figure 6 for the case when the load speed is $u=8.9 \mathrm{~m} / \mathrm{s}$ In particular it should be noted that the contours shown in the two-dimensional wave pattern in the left panel of Figure 6 are of very small amplitude. Examining the center line of the wave deflection in the right panel, it becomes clear that the shadow zone discovered by Davys, Hosking \& Sneyd (1985) using the linear steady model is recovered in our nonlinear time-dependent model.

In the field campaign of Wilson (1955), an ammunition truck was driven over the ice-covered Mille Lacs near Brainerd, Minnesota in the United States of America. The deflection of the ice cover was measured with a deflectometer, the depth at the measuring point was about $3.26 \mathrm{~m}$, and the ice thickness was $0.61 \mathrm{~m}$. The elastic modulus was estimated to be $9 \times 10^{9} \mathrm{~N} / \mathrm{m}^{2}$ (see Table 1). The non-dimensional damping coefficient was taken to be $B=0.95$. Figure 7 shows comparisons with these experimental data. The load speeds (from top to bottom) are $2.6 \mathrm{~m} / \mathrm{s}, 4.6 \mathrm{~m} / \mathrm{s}, 7.3 \mathrm{~m} / \mathrm{s}, 8.4 \mathrm{~m} / \mathrm{s}$, and $17.9 \mathrm{~m} / \mathrm{s}$ The data are compared to numerical approximations of solutions to the two-dimensional model (5.3), (5.4). The match between experimental data and numerical approximation is superb for the two subcritical cases. For the third and fourth case, the match is not as good, but given a probably rather large uncertainty in the measurements, the comparison is overall fair.

Figure 8 shows comparisons with the experimental data of Wilson (1955) pertaining to the two-truck experiments. In these runs, two trucks were driven over the ice at a fixed distance. The comparison with the numerical approximation of $(5.3),(5.4)$ is quite good, and in particular better than the single-truck case.

Lastly, we consider the experimental work of Beltaos (1981) carried out on Cold Lake which is located on the Alberta-Saskatchewan provincial boundary in Canada. A truck was driven along a test track located about $800 \mathrm{~m}$ from shore. The water depth was about $4.3 \mathrm{~m}$ and the ice thickness was $0.59 \mathrm{~m}$. The elastic modulus was estimated to be $4.9 \times 10^{9} \mathrm{~N} / \mathrm{m}^{2}$ (see Table 1 ). Note that while the dispersion curve also has two critical values in this case, they are so close together that in practice there is only one critical load speed, which Beltaos determined experimentally to be about $V_{c}=7.3 \mathrm{~m} / \mathrm{s}$.

Figure 9 shows comparisons of the numerical approximations of (5.3), (5.4) with the experimental data of Beltaos (1981). The load speeds (from top to bottom) are $4.4 \mathrm{~m} / \mathrm{s}$, $8.0 \mathrm{~m} / \mathrm{s}$ and $13.9 \mathrm{~m} / \mathrm{s}$. The first load speed is subcritical, while the second and third are supercritical. The comparison of the wave profile is qualitatively good, but the numerical simulation slightly underpredicts the maximum deflection of the ice sheet. Of course, as also pointed out by Beltaos (1981), there is considerable uncertainty in the measurements, 

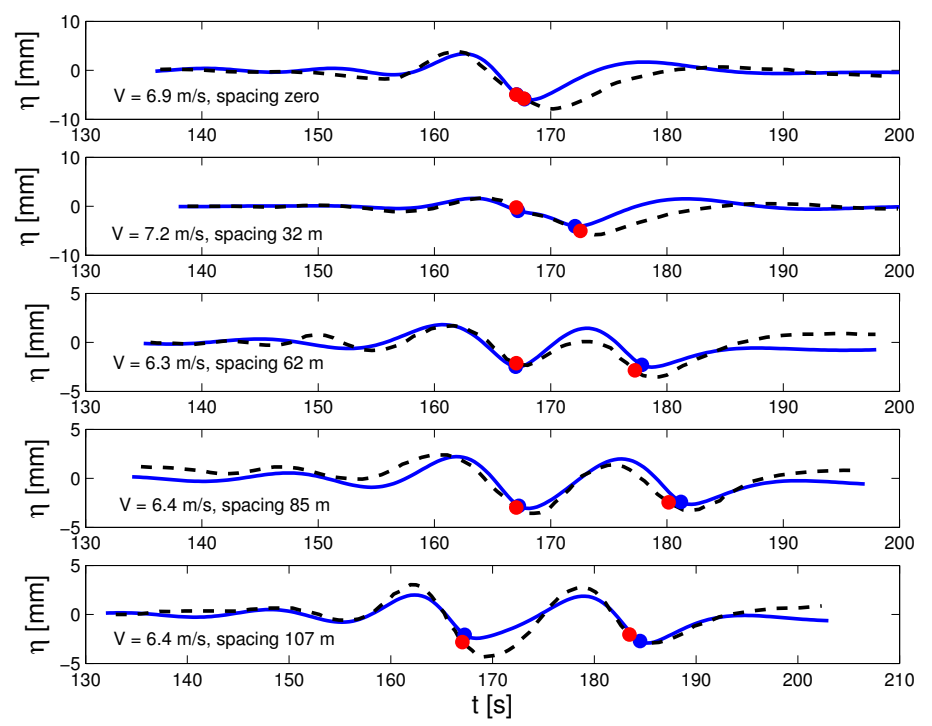

FiguRE 8. Comparison of numerical approximation of equations (5.3), (5.4) and experimental ice deflection records with two trucks driving at constant speed. The dashed black curves are the experimental data of Wilson (1955), and the red dots indicate the $z$-position of the two trucks used in these experiments at the time they pass the deflectometer. The blue curves represent the a time series of the numerical approximation of equations (5.3), (5.4), taken at a measuring station corresponding to the position of the deflectometer in the field experiment. The blue dots represent the $z$-position position of the two loads as they pass the position where the time series is obtained.

and as in the cases above, the damping parameter has to be estimated based on the fit with the experiments. In this case, the non-dimensional damping coefficient was taken to be $B=1.05$. For the near-critical case with load speed $V=1.1 V_{c}=8.0 \mathrm{~m} / \mathrm{s}$ the two-dimensional wavecrest pattern is shown in Figure 10. Since the load speed is above the critical speed, one also expects a shadow zone in this case, and this can bee seen in the wavecrest pattern in the left panel of Figure 10, and in the centerline deflection in the right panel. The small trailing disturbances are transients which decay over time.

Beltaos (1981) also raised the interesting question of whether a changing load speed may have an impact on the response of the ice sheet. In particular, he noted that drivers have reported ice failures occurring in the case of sudden deceleration because of a perceived danger ahead. As will be shown presently, deceleration may indeed have an adverse effect on the stability of the ice sheet, as interference patterns may contribute to exceeding the critical strain beyond which failures are likely to occur.

Figure 11 shows the development of the free surface for a load passing a measuring station. The left panels show a load moving at constant speed. The curve in the upper left panel depicts the deflection of the ice sheet at the measuring station, and the black dot indicates the time when the load passes the measuring station. The middle left panel shows the vertical position of the free surface at the point of the load, and the lower left panel shows the maximum strain of the ice. The strain computed here is the linear or axial strain which is used in defining the bending moment of an elastic solid, and which can be measured experimentally using a strainmeter, such as explained in Davys, Hosking \& Sneyd (1985). According to Page \& Părău (2014) and references therein, the strain is approximated by the expression $\varepsilon=\frac{h}{2} \eta_{x x}$. The strain takes on large values at the beginning which is probably due to the impulsively started load (even though we 

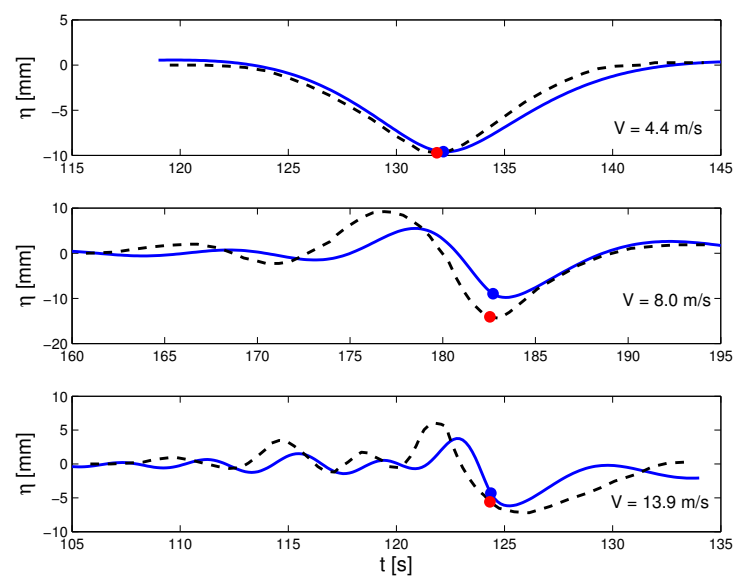

Figure 9. Comparison of numerical approximation of equations (5.3), (5.4) and experimental ice deflection records. The dashed black curves are the experimental ice deflection records of Beltaos (1981), and the red dots indicate the $z$-position of the truck as it passes the deflectometer. The blue curves represent time series of the numerical approximation of equations (5.3), (5.4), taken at a measuring point corresponding to the position of the deflectometer in the field experiments. The blue dots represent the $z$-position of the numerically imposed load as it passes the measuring point.
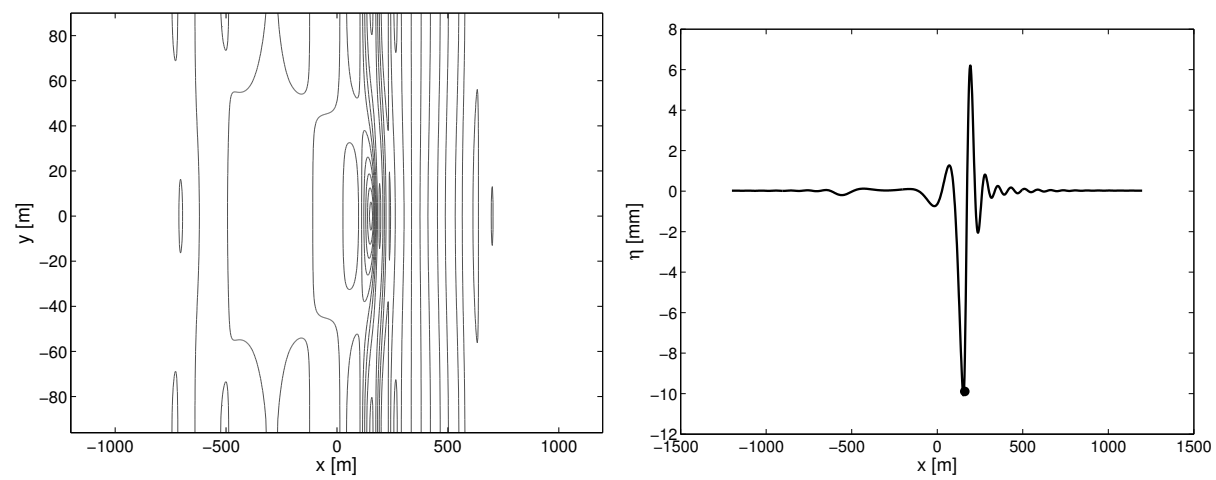

FiguRE 10. Numerical approximation of equations (5.3), (5.4) for constant load velocity $V=8.0 \mathrm{~m} / \mathrm{s}$, such as in Figure 9. The left panel shows the wave crest pattern, and the right panel shows the deflection of the ice sheet along the center line $y=0$.

made sure to use initial data corresponding to a static load so as to avoid a non-smooth start-up phase). Otherwise the maximum strain is on the order of $10^{-5}$.

The panels on the right in Figure 11 show the case of a decelerating load. The upper right panel shows the development of the surface deflection at the measuring station, and the black dot indicates the time when the load passes the measuring station. We note that the surface deflection is larger by a factor of more than 10 compared to the case of a load moving at constant speed. The middle right panel shows the corresponding vertical position of the load on the ice sheet, and the amplitude here is also larger by a factor of 10 when compared to the case of constant propagation speed of the load. Finally, the lower right panel shows the maximum strain in the ice sheet as a function of time. Here it must be noted that the maximum strain occurring at time $t=28$ is about $2 \times 10^{-4}$ which is approaching the value $2.14 \times 10^{-4}$ which was indicated in Goodman, Wadhams \& Squire (1980) as the critical strain where cracks may appear in the ice. 

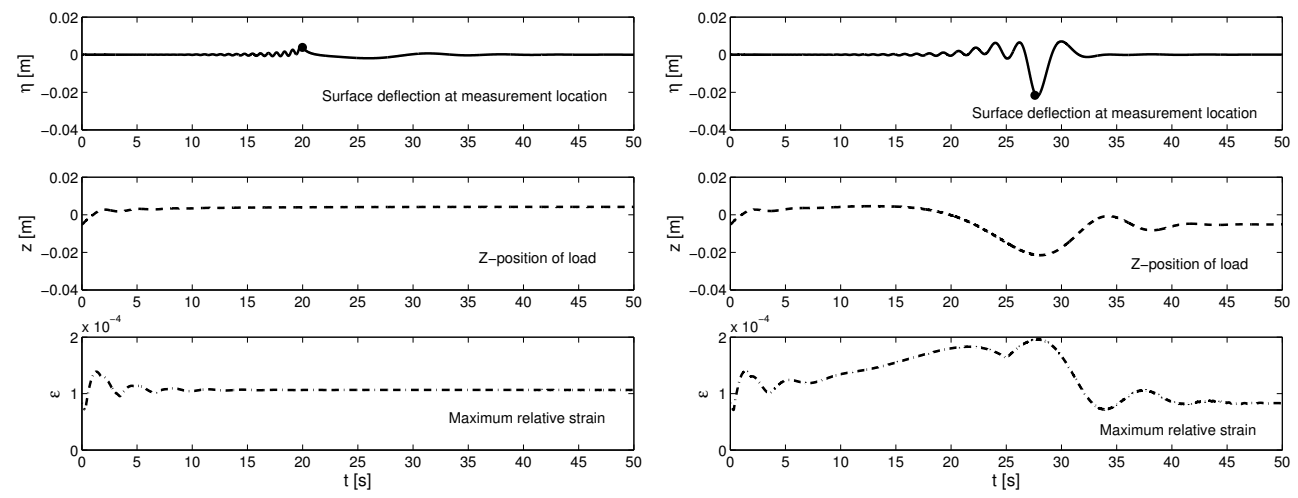

Figure 11. Comparison of numerical approximation of equations (4.6), (4.12) for constant load velocity $10 \mathrm{~m} / \mathrm{s}$ (left) and deceleration from $10 \mathrm{~m} / \mathrm{s}$ to $0 \mathrm{~m} / \mathrm{s}$ (right). The upper panels show the evolution of the surface deflection at a measuring station at a fixed location. The middle panels show the $z$ position of the load as a function of time. The lower panels show the maximum relative strain as a function of time.
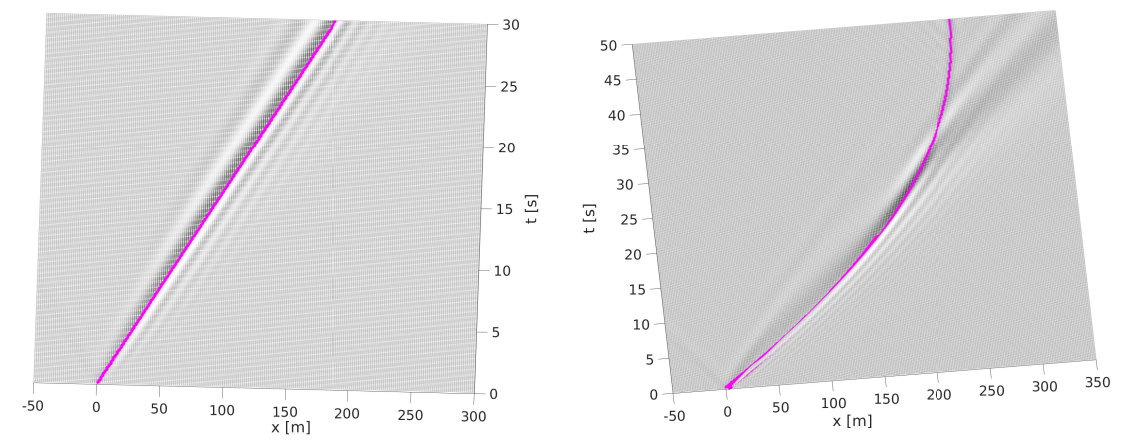

FiguRE 12. Comparison of numerical approximation of equations (4.6), (4.12) for constant load velocity $6.2 \mathrm{~m} / \mathrm{s}$ (left) and deceleration from $10 \mathrm{~m} / \mathrm{s}$ to $0 \mathrm{~m} / \mathrm{s}$ (right). The position of the load is indicated in magenta. The left panel shows gravity waves trailing the load, while the right panel show gravity waves continuously catching up with the load.

Maximum ice deflection and strain are summarized in Table 2. Note that the values of maximum strain are slightly decreasing as the deceleration gets higher. From this trend, one may conclude that it is actually safer to brake swiftly rather than to brake slowly. The dependence of the maximum deflection and maximum strain on the load appears to be approximately linear.

The stark difference between the cases of constant load speed and decelerating load may be explained by constructive interference of waves of different phase speed created by the changing speed of the load. Indeed, as the load decelerates through the critical longwave speed $\sqrt{g H}$, it continuously excites gravity wave of smaller and smaller velocities. The analysis in Takizawa (1987) shows that gravity waves will generally trail the moving load, but as the load velocity is getting smaller than the previously excited waves, the waves immediately catch up with the load, and through constructive interference an increasing bulge and trough traveling with the load is formed. Eventually, this leads to much larger surface deflections than could be expected with constant load speed. The time development of these two cases are contrasted in Figure 12. 


$\begin{array}{lccccc}\text { Deceleration rate } & 0.2 & 0.4 & 0.6 & 0.8 & \mathrm{~m} / \mathrm{s}^{2} \\ \text { Max. deflection } & 0.022 & 0.018 & 0.016 & 0.015 & \mathrm{~m} \\ \text { Max. strain } & 1.97 \mathrm{e}-04 & 1.76 \mathrm{e}-04 & 1.61 \mathrm{e}-04 & 1.51 \mathrm{e}-04 & \end{array}$

TABle 2. Maximum ice deflection and maximum strain rate during the deceleration of a light vehicle. The parameter values for this study have been taken from the experiments by Takizawa (1987), summarized in Table 1.

\section{Conclusions}

Following Whitham's idea, we have developed a fully dispersive weakly nonlinear system describing flexural-gravity waves in an elastic plate on a fluid foundation, excited by a moving load. The system is written in terms of the deflection of the elastic plate and the surface trace of the fluid velocity potential. The system derived here gives an accurate description of the linearized dynamics as the full linear dispersion relation is incorporated in the systems. The system also allows for multi-directional wave propagation and various configurations of the imposed load.

The equations are also nonlinear which makes the model more flexible in the case when shallow fluids or very thin elastic plates are to be described. Indeed, nonlinearity may be a desirable feature of a model for hydro-elastic responses as there are observations of largeamplitude hydro-elastic waves in coastal regions. For example, such waves were described in Marko (2003). Note also that Liu \& Mollo-Christensen (1988) describe focussing of wave energy in an ice pack which can lead to modulational instability and large-amplitude waves.

The system has been approximated numerically, and the results have been compared with a few experimental measurements. Overall, fairly good agreement with the available experimental measurements has been obtained. Both the one-dimensional model (4.6), (4.12) and the one-dimensional model (5.3), (5.4) have been used in the simulations. In general, it appears that as long as the load configuration is such that a point mass may be used to model the load, there is no particular advantage in using the two-dimensional equations (5.3), (5.4) for making predictions from a practical point of view. Of course, if the precise nature of the wave pattern is under study then the two-dimensional model needs to be used. The two-dimensional model will also have to be used if the load configuration is such that strongly two-dimensional wave patterns are to be expected, such as the motion of a train on tracks laid on an ice sheet for example.

We have also investigated the question of variable load speed, and we have found that under certain conditions, the wave amplitude during decelerating motion can exceed the corresponding values due to constant speed by a large factor. Indeed, for otherwise reasonable parameter values, the maximum strain may approach values where ice breakup may occur. Future work may focus on studying a larger number of cases with a particular focus on non-constant load velocities in order to identify potentially hazardous configurations.

\section{Appendix A. Comparison with a potential model}

In Section 8, the fully dispersive damped model was compared with field experiments. Due to safety concerns, ice break-up must be avoided in these experiments. As a consequence, these data do not feature large icesheet deflections, and the role of nonlinearity in the equations is of minor importance. We nevertheless incorporated weakly nonlinear terms into the equations since large surface deflections are known to occur. In order to 


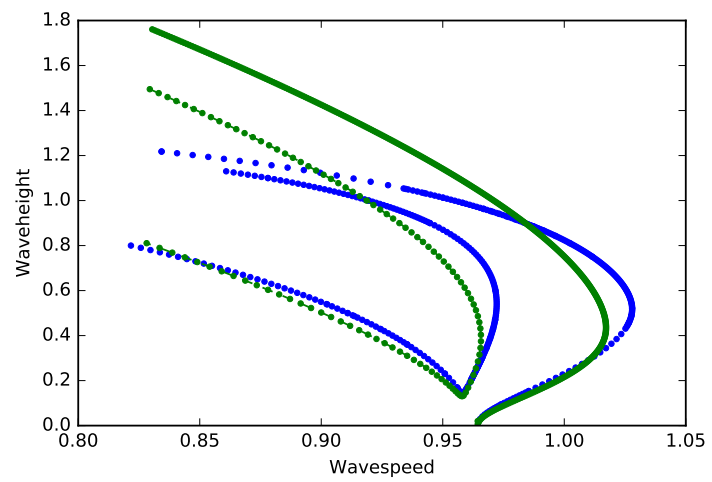

FIgURE 13. Bifurcation diagrams for $\lambda=4 \pi$ and $\tau=0.1$. The blue curve represents solutions of the hydro-elastic full Euler system. The green curve represents solutions of the fully dispersive weakly nonlinear equation (1.1)

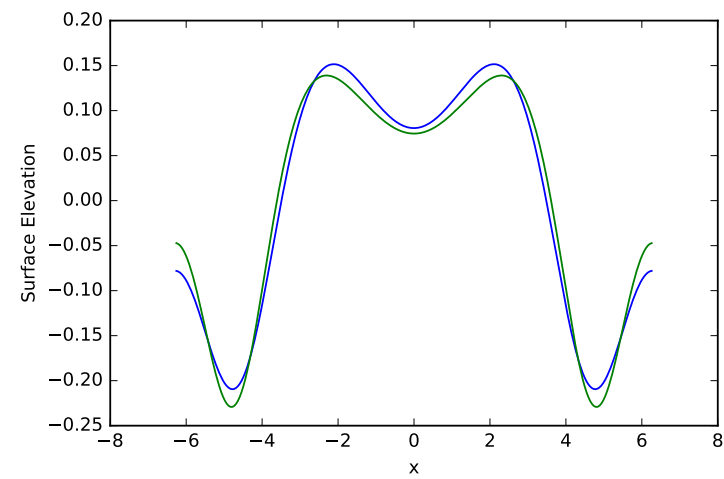

Figure 14. Solutions profiles for $\lambda=4 \pi$ and $\tau=0.1$. The blue curve is an approximate solutions of the hydro-elastic full Euler system with wave speed $c=0.9353$. The green curve is an approximate solution of the Whitham equation with $c=0.9257$.

verify that the equations also give representative results in the case of larger amplitudes, we now analyze the weakly nonlinear approximation by comparing with solutions of the full Euler system.

In order to focus on a concrete situation, we consider traveling-wave solutions in the case without an external load. In addition, we will assume that the ice is thin, so that $h=0$, and damping is negligible, i.e. $b=0$. These are standard assumptions which are known to lead to the existence of traveling waves for the full Euler system Plotnikov \& Toland (2011). The fully nonlinear Euler equations can be solved numerically by applying a conformal mapping technique.

Taking the depth $H$ as a unit of length and the long-wave speed $\sqrt{g H}$ as a unit of velocity, one may rewrite the complete system in non-dimensional form. Omitting details and referring the reader to Guyenne \& Părău (2014a) we only note that the Bernoulli equation takes the form

$$
\phi_{t}+\frac{1}{2}\left(\phi_{x}^{2}+\phi_{z}^{2}\right)+\eta+\tau\left(\kappa_{s s}+\frac{1}{2} \kappa^{3}\right)=0 \quad \text { for } \quad x \in \mathbb{R}, \quad z=\eta(x, t),
$$


where $\kappa=\eta_{x x}\left(1+\eta_{x}^{2}\right)^{-3 / 2}$ is the curvature of the shell and $s$ is the arclength along this cover and so

$$
\kappa_{s s}+\frac{1}{2} \kappa^{3}=\frac{1}{\sqrt{1+\eta_{x}^{2}}} \partial_{x}\left(\frac{1}{\sqrt{1+\eta_{x}^{2}}} \partial_{x}\left(\frac{\eta_{x x}}{\left(1+\eta_{x}^{2}\right)^{3 / 2}}\right)\right)+\frac{1}{2}\left(\frac{\eta_{x x}}{\left(1+\eta_{x}^{2}\right)^{3 / 2}}\right)^{3} .
$$

In our framework of Kirchhoff-Love assumptions the latter simplifies to the form

$$
\kappa_{s s}+\frac{1}{2} \kappa^{3}=\partial_{x}^{4} \eta
$$

however, for technical reasons we use the exact expression in our calculations in the case of the full Euler system. The hydro-elastic parameter $\tau=\mathcal{D} /\left(\rho g H^{4}\right)=\varkappa / H^{4}$ is now non-dimensional. We approximate travelling wave solutions numerically, following the method laid out in Guyenne \& Părău (2014a) and compare them with ones for the Whitham equation (1.1).

We set the hydro-elastic parameter $\tau=0.1$, the wavelength $\lambda=4 \pi$ and construct periodic solutions on the interval $[-\lambda / 2, \lambda / 2]$. The choice of $\tau$ and $\lambda$ seems reasonable as these values correspond, for example, to a depth $H \approx 4$ meters and a wavelength $L=\lambda H \approx 50$ meters which appear physically reasonable.

We observe solutions of two types in these settings. There are solutions bifurcating from trivial linear solutions. They can be approximated asymptotically by linear theory, at least if the amplitudes are not too high. There are, however, solutions that cannot be predicted or approximated by linear theory at all (the leftmost branch in Figure 13). They start to appear when the non-dimensional waveheight reaches approximately 0.14 according to Figure 13. Thus it can be inferred that waves of waveheight $56 \mathrm{~cm}$ in the case of a depth of $H=4 \mathrm{~m}$ could be purely nonlinear in nature. Notably, as seen in Figure 14 weakly nonlinear modelling, i. e. use of the Whitham equation, can give high accuracy up to moderate waveheights.

Acknowledgments. This research was supported by the Research Council of Norway through grant no. 239033/F20, E.I. Părău has been partially supported by the EPSRC under grant EP/J019305/1. The authors would like to thank the Isaac Newton Institute for Mathematical Sciences, Cambridge, for support and hospitality during the programme Mathematics of sea ice phenomena where work on this paper was undertaken (EPSRC grant no EP/K032208/1 and Simons Foundation).

\section{REFERENCES}

Aceves-Sánchez, P., Minzoni, A. A. \& Panayotaros, P. 2013 Numerical study of a nonlocal model for water-waves with variable depth. Wave Motion 50, 80-93.

Babaei, H., Van der Sanden, J., Short, N. \& Barrette, P. 2016 Lake ice cover deflection induced by moving vehicles: comparing theoretical results with satellite observations. In TAC 2016: Efficient Transportation-Managing the Demand-2016 Conference and Exhibition of the Transportation Association of Canada.

Beltaos, S. 1981 Field studies on the response of floating ice sheets to moving loads. Canadian J. Civil Engineering 8, 1-8.

Bonnefoy, F., Meylan, M. H. and Ferrant, P. 2009 Nonlinear higher-order spectral solution for a two-dimensional moving load on ice. J. Fluid Mech. 621 (2009), 215-242.

CARTER, J. D. 2018 Bidirectional Whitham equations as models of waves on shallow water. Wave Motion 82, 51-61.

Craig, W. And Groves, M. D. 1994 Hamiltonian long-wave approximations to the water-wave problem. Wave Motion 19 (1994), 367-389. 
Craig, W., Guyenne, P. \& Kalisch, H. 2005 Hamiltonian long-wave expansions for free surfaces and interfaces. Comm. Pure Appl. Math. 58, 1587-1641.

Craig, W. And Sulem, C. 1993 Numerical simulation of gravity waves. J. Comp. Phys. 108, $73-83$.

Davys, J. W. Hosking, R. J. \& Sneyd, A. D. 1985 Waves due to a steadily moving source on a floating ice plate. J. Fluid Mech. 158, 269-287.

Dinvay, E., Dutykh, D. \& Kalisch, H. 2019 A comparative study of bi-directional Whitham systems. Appl. Num. Math. 141, 248-262.

Dinvay, E., Moldabayev, D., Dutykh, D. \& Kalisch, H. 2017 The Whitham equation with surface tension. Nonlinear Dynamics 88, 1125-1138.

Ehrnström, M. \& Kalisch, H. 2009 Traveling waves for the Whitham equation. Diff. Int. Eq. 22, 1193-1210.

Ehrnström, M. \& Kalisch, H. 2013 Global bifurcation for the Whitham equation. Math. Modeling Natural Phenomena 8, 13-30.

Goodman, D. J., Wadhams, P. \& Squire, V. A. 1980 The flexural response of a tabular ice island to ocean swell. Ann. Glaciol. 1, 23-27.

Guyenne, P. \& PĂRĂU, E. I. 2012 Computations of fully-nonlinear hydroelastic solitary waves on deep water. J. Fluid Mech. 713, 307-329.

Guyenne, P. \& PĂRĂU, E. I. 2014a Finite-Depth Effects on Solitary Waves in a Floating Ice-Sheet. J. Fluids and Structures 49, 242-262.

GuYenne, P. \& PĂRĂU, E. I. 2014b Forced and unforced flexural-gravity solitary waves. Proc. IUTAM 11, 44-57.

GUYENnE, P. \& PĂRĂU, E. I. 2017 Numerical simulation of solitary-wave scattering and damping in fragmented sea ice, Proc. 27th International Ocean and Polar Engineering Conference (ISOPE 2017), pp. 373-380, (2017).

Hosking, R. J., Sneyd, A. D. And Waugh, D. W. 1988 Viscoelastic response of a floating ice plate to a steadily moving load. J. Fluid Mech. 196, 409-430.

Hur, V. M. \& Johnson, M. 2015a Modulational instability in the Whitham equation for water waves. Stud. Appl. Math. 134, 120-143.

Hur, V. M. \& Johnson, M. 2015b Modulational instability in the Whitham equation with surface tension and vorticity. Nonlinear Anal. 129, 104-118.

Kheysin, D. Ye. 1963 Moving load on an elastic plate which floats on the surface of an ideal fluid (in Russian). Izv. AN USSR, Otd, Tekh. i Mashinostroenie 1, 178-180.

Kheysin, D. Ye. 1971 Some unsteady-state problems in ice-cover dynamics. In Studies in Ice Physics and Ice Engineering (ed. G. N. Yakovlev, pp. 69-78. Israel Program for Scientific Translations.

Lannes, D. AND SAut, J.-C. 2013 Remarks on the full dispersion Kadomtsev-Petviashvli equation. Kinet. Relat. Models 6, 989-1009.

Liu, A. K. \& Mollo-Christensen, E. 1988 Wave propagation in a solid ice pack. J. Phys. Oceanogr. 18, 1702-1712.

Madsen, P. A., Murray, R. \& Sørensen, O. R. 1991 A new form of the Boussinesq equations with improved linear dispersion characteristics. Coastal Engineering 15, 371-388.

Marchenko, A. V. 1988 Long waves in shallow liquid under ice cover. J. Appl. Math. Mech. 52, 180-183.

Marchenko, A. V. 2016 Damping of surface waves propagating below solid ice. In The 26th International Ocean and Polar Engineering Conference, ISOPE.

MARKo, J. R. 2003 Observations and analyses of an intense waves-in-ice event in the Sea of Okhotsk. J. Geoph. Res. 108, 3296.

Matiushina, A. A., Pogorelova, A. V. And Kozin, V. M. 2016 Effect of impact load on the ice cover during the landing of an airplane. Internat. J. Offshore Polar Engnrng. 26, $6-12$.

Miles, J. \& Sneyd, A. D. 2003 The response of a floating ice sheet to an accelerating line load. J. Fluid Mech. 497, 435-439.

Milinazzo, F., Shinbrot, M. And Evans, N. W. 1995. A mathematical analysis of the steady response of floating ice to the uniform motion of a rectangular load. J. Fluid Mech. 287, 173-197. 
Moldabayev, D., Kalisch, H. \& Dutykh, D. 2015 The Whitham Equation as a model for surface water waves. Phys. D 309, 99-107.

Nevel, D. E. 1970 Moving loads on a floating ice sheet. CRREL Res. Rep. 261, US Army Cold Regions Research and Engineering Laboratory, Hanover, NH, USA.

Nicholls, D. P. \& Reitich, F. 2001 A new approach to analyticity of Dirichlet-Neumann operators. Proc. Roy. Soc. Edinburgh Sect. A 131, 1411-1433.

Nugroho, W. S., Wang, K., Hosking, R. J. \& Milinazzo, F. 1999 Time-dependent response of a floating flexible plate to an impulsively started steadily moving load. J. Fluid Mech. 381, 337-355.

Nwogu, O. 1993 Alternative form of Boussinesq equations for nearshore wave propagation. Journal of Waterway, Port, Coastal, Ocean Engineering 119, 618-638.

PAge, C. AND PĂRĂU, E. I. 2014 Hydraulic falls under a floating ice plate due to submerged obstructions. J. Fluid Mech. 745, 208-222.

PĂRĂU, E. I. \& VANDEN-Broeck, J.-M. 2011 Three-dimensional waves beneath an ice sheet due to a steadily moving pressure. Phil. Trans. Roy. Soc. A 369, 2973-2988.

PĂRĂU, E. I. \& DIAS, F. 2002 Nonlinear effects in the response of a floating ice plate to a moving load. J. Fluid Mech. 460, 281-305.

Plotnikov, P. I. \& Toland, J. F. 2011 Modelling nonlinear hydroelastic waves. Phil. Trans. R. Soc. A 369, 2942-2956;

Pogorelova, A. V. 2008 Wave resistance of an air-cushion vehicle in unsteady motion over an ice sheet. J. Appl. Mech. Tech. Phys. 49, 71-79.

Sanford, N., Kodama, K., Carter, J. D. \& Kalisch, H. 2014 Stability of traveling wave solutions to the Whitham equation. Phys. Lett. A 378, 2100-2107.

Schulkes, R. M. S. M. \& Sneyd, A. D. 1988 Time-dependent response of floating ice to a steadily moving load. J. Fluid Mech. 186, 25-46.

Squire, V. A., Robinson, W. H., Langhorne, P. J. \& Haskell, T.G. 1988 Vehicles and aircraft on floating ice. Nature 333, 159-161.

Squire, V. A., Hosking, R. J., Kerr, A. D. \& Langhorne, P. J. 1988 Moving Loads on Ice Plates. Kluwer, Dordrecht.

TAKIZAWA, T. 1978 Deflection of a floating ice sheet subjected to a moving load II (in Japanese). Low Temperature Science A 37, 69-78.

TAkizawa, T. 1985 Deflection of a floating sea ice sheet induced by a moving load. Cold Regions Science and Technology 11, 171-180.

TAKizAwA, T. 1987 Field studies on response of a floating sea ice sheet to a steadily moving load. Contrib. Inst. Low Temp. Sci. A 36, 31-76.

TAKIZAWA, T. 1988 Response of a floating sea ice sheet to a steadily moving load. J. Geophys. Res. 93, 5100-5112.

VAn der SAnden, J. \& Short, N. H. 2017 Radar satellites measure ice cover displacements induced by moving vehicles. Cold Regions Science and Technology 133, 56-62.

Vargas-Magana, R.M. \& Panayotaros, P. 2016 A Whitham-Boussinesq long-wave model for variable topography. Wave Motion 65, 156-174.

Wang, K., Hosking, R. J. \& Milinazzo, F. 2004 Time-dependent response of a floating viscoelastic plate to an impulsively started moving load. J. Fluid Mech. 521, 295-317.

Wei, G., Kirby, J. T., Grilli, S. T. \& Subramanya, R. 1995 A fully nonlinear Boussinesq model for surface waves. Part 1. Highly nonlinear unsteady waves. J. Fluid Mech. 294, $71-92$.

Whitham, G. B. 1967 Variational methods and applications to water waves. Proc. Roy. Soc. London A 299, 6-25.

Whitham, G. B. 1972 Linear and Nonlinear Waves (Wiley, New York, 1974).

Wilson, J.T. 1955 Coupling between moving loads and flexural waves in floating ice sheets. US Army SIPRE Report 34.

XIA, X. \& Shen, H. T. 2002 Nonlinear interaction of ice cover with shallow water waves in channels. J. Fluid Mech. 467, 259-268. 